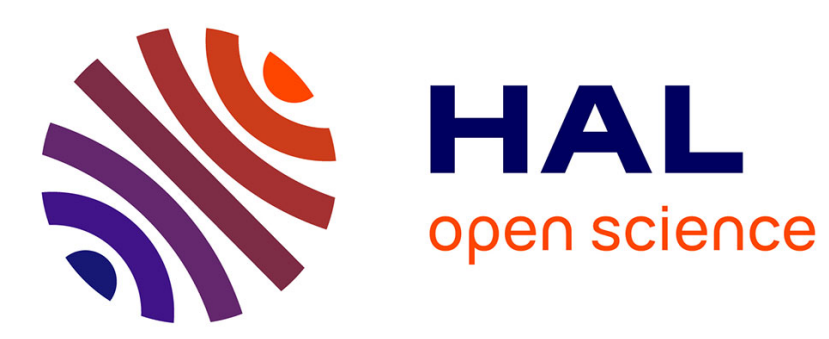

\title{
Error estimate for Godunov approximation of locally constrained conservation laws
}

Clément Cancès, Nicolas Seguin

\section{To cite this version:}

Clément Cancès, Nicolas Seguin. Error estimate for Godunov approximation of locally constrained conservation laws. SIAM Journal on Numerical Analysis, 2012, 50 (6), pp.3036-3060. $10.1137 / 110836912$. hal-00599581v2

\section{HAL Id: hal-00599581 \\ https://hal.science/hal-00599581v2}

Submitted on 1 Mar 2012

HAL is a multi-disciplinary open access archive for the deposit and dissemination of scientific research documents, whether they are published or not. The documents may come from teaching and research institutions in France or abroad, or from public or private research centers.
L'archive ouverte pluridisciplinaire $\mathbf{H A L}$, est destinée au dépôt et à la diffusion de documents scientifiques de niveau recherche, publiés ou non, émanant des établissements d'enseignement et de recherche français ou étrangers, des laboratoires publics ou privés. 


\title{
Error estimate for Godunov approximation of locally constrained conservation laws
}

\author{
Clément CanCES* Nicolas Seguin ${ }^{\dagger}$
}

March 1, 2012

\begin{abstract}
We consider a model of traffic flow with unilateral constraint on the flux introduced by R. M. CoLOmbo and P. Goatin (J. Differ. Equ. 234(2):654-675, 2007), for which the convergence of numerical approximation using monotone finite volume schemes has been performed by B. ANDREIANOV et al. (Numer. Math. 115:609-645, 2010). We derive for this problem some new BV-estimate, and make use of it to provide an error estimate for the Godunov approximation of the problem of order $h^{1 / 3}$ that is improved into the optimal order $h^{1 / 2}$ under a reasonable assumption. Numerical experiments are then provided to illustrate the optimality of the result.
\end{abstract}

keywords: Locally constrained scalar conservation laws, monotone finite volume scheme, BV-estimate, error estimate.

AMS 35L65, 65M15, 76M12, 90B20

\section{Presentation of the continuous problem}

Recently, R. M. Colombo and P. Goatin [14] analyzed the following model of traffic flow, inspired from the so-called LWR model [21, 25], with a constraint on the flux:

$$
\begin{cases}\partial_{t} u+\partial_{x} f(u)=0 & \text { for }(x, t) \in \mathbb{R} \times \mathbb{R}_{+}, \\ u(x, 0)=u_{0}(x) & \text { for } x \in \mathbb{R} \\ f(u)(0, t) \leq F(t) & \text { for } t \in \mathbb{R}_{+}\end{cases}
$$

where $f$ is supposed to be Lipschitz continuous on $[0,1]$ and bell-shaped, i.e. there exists $\bar{u} \in(0,1)$ such that

$$
f(0)=f(1)=0, \quad f \geq 0, \quad f^{\prime}(u)(\bar{u}-u)>0 \text { for a.e. } u \in[0,1],
$$

and where the constraint $F$ satisfies

$$
0 \leq F(t) \leq f(\bar{u}) \text {, for a.e. } t \in \mathbb{R}_{+} .
$$

This constraint $F$ models toll gates or traffic lights. In the case where the flow is not constrained, i.e. $F \equiv f(\bar{u})$, then it is well known that the good notion of solution for the problem (1) is the notion of entropy solution $[23,28,19]$. In the case where the constraint becomes active, i.e. $F(t)<f(\bar{u})$ on a non negligible set of $\mathbb{R}_{+}$, then it is shown in [14] that a non-classical shock with zero speed can appear at the interface $\{x=0\}$, so that the constraint can be satisfied. It has then been pointed out by B. Andreianov, P. GoAtin and N. SEguin [4] that the problem (1) can be immersed in the framework of scalar conservation laws with discontinuous flux functions, that has been widely studied during the last years. Among the numerous references available on this topic, let us mention that AdimurThi, S. Mishra and G.D. Veerappa GOwDA [1] exhibited that such scalar conservation laws with discontinuous flux function admit an infinite number of solutions.

*UPMC Univ Paris 06 \& CNRS, UMR 7598, Laboratoire Jacques-Louis Lions, F-75005, Paris (cances@ann.jussieu.fr)

†UPMC Univ Paris 06 \& CNRS, UMR 7598, Laboratoire Jacques-Louis Lions, F-75005, Paris (nicolas.seguin@upmc.fr) 
More precisely, we look for solutions of

$$
\begin{cases}\partial_{t} u+\partial_{x} f(u)=0 & \text { for }(x, t) \in \mathbb{R} \times \mathbb{R}_{+}, \\ u(x, 0)=u_{0}(x) & \text { for } x \in \mathbb{R},\end{cases}
$$

that satisfy the usual entropy criterion away from the interface, i.e. such that

$$
\partial_{t}|u-\kappa|+\partial_{x} \Phi_{\kappa}(u) \leq 0, \text { in } \mathcal{D}^{\prime}\left([0,+\infty) \times \mathbb{R}_{+}\right),
$$

where

$$
\Phi_{\kappa}(u)=\operatorname{sign}(u-\kappa)(f(u)-f(\kappa)) .
$$

We also require the continuity of the flux at $\{x=0\}$, yielding the Rankine-Hugoniot condition

$$
f\left(u_{L}\right)=f\left(u_{R}\right),
$$

where $u_{L}$ and $u_{R}$ respectively denote the one-sided traces of $u$ on $\{x=0\}$ from $\{x<0\}$ and $\{x>0\}$, i.e.

$$
u_{L}(t)=\lim _{\epsilon \rightarrow 0^{+}} \frac{1}{\epsilon} \int_{-\epsilon}^{0} u(x, t) d x, \quad u_{R}(t)=\lim _{\epsilon \rightarrow 0^{+}} \frac{1}{\epsilon} \int_{0}^{\epsilon} u(x, t) d x .
$$

Note that, following E. YU. PANOv [24], since the flux function $f$ is non constant on any interval and since the solution $u$ satisfies (4), then the traces $u_{L, R}$ defined by (7) exist for almost all $t>0$.

As it was first noticed in [1], uniqueness of the solution of (3)-(6) fails since some undercompressive shocks can be generated by the interface $\{x=0\}$, yielding an infinite number of solutions. More precisely, define

$$
\mathcal{U}=\left\{(A, B) \in[0,1]^{2} \mid f(A)=f(B), A \geq \bar{u} \text { and } B \leq \bar{u}\right\}
$$

the set of all the possible stationary undercompressive shocks. For all $(A, B) \in \mathcal{U}$, the piecewise constant function

$$
x \mapsto A \mathbb{1}_{x<0}(x)+B \mathbb{1}_{x>0}(x)
$$

is a steady solution to the problem (3)-(6), being a usual entropy solution in the sense of [19, 23] if and only if $A=B=\bar{u}$. It has been emphasized in [5] that for all $(A, B) \in \mathcal{U}$, there exists a unique $L^{1}$-contraction semi-group such that (8) is a solution to the problem. Reciprocally, given a time independent $L^{1}$-contraction semi-group for the problem (3)-(6), there exists a unique $(A, B) \in \mathcal{U}$ such that (8) is a solution to the problem. Let $u$ be the solution of (3)-(6) belonging to this latter $L^{1}$-contraction semi-group, then its one-sided traces satisfy

$$
\Phi_{B}\left(u_{R}(t)\right)-\Phi_{A}\left(u_{L}(t)\right) \leq 0, \quad \text { for a.e. } t>0 .
$$

Moreover, it has been pointed out in [3] that

$$
f\left(u_{L}\right)=f\left(u_{R}\right) \leq f(A)=f(B) \quad \text { for a.e. } t>0,
$$

so that the choice of $(A, B) \in \mathcal{U}$ enforces a constraint on the flux at the interface.

Symmetrically, let $F \in L^{\infty}\left(\mathbb{R}_{+} ;[0, f(\bar{u})]\right)$, then there exists a unique couple $(A, B) \in\left(L^{\infty}\left(\mathbb{R}_{+} ;[0,1]\right)\right)^{2}$ such that

$$
A(t) \geq \bar{u} \quad(\text { resp. } B(t) \leq \bar{u}), \quad F(t)=f(A(t))=f(B(t)) .
$$

Obviously, $(A(t), B(t)) \in \mathcal{U}$ for a.e. $t>0$.

Definition 1.1 Let $F \in L^{\infty}\left(\mathbb{R}_{+} ;[0, f(\bar{u})]\right)$, and let $A, B \in L^{\infty}\left(\mathbb{R}_{+}\right)$be defined almost everywhere by (9), then the subset $\mathcal{G}^{*}(t)$ of $[0,1]^{2}$, defined by: for a.e. $t>0$,

$$
\mathcal{G}^{*}(t)=\left\{\left(c_{L}, c_{R}\right) \in[0,1]^{2} \mid f\left(c_{L}\right)=f\left(c_{R}\right) \text { and } \Phi_{B(t)}\left(c_{R}\right)-\Phi_{A(t)}\left(c_{L}\right) \leq 0\right\} .
$$

is said to be the $L^{1}$-dissipative (dual) germ corresponding to $F$.

We refer to [5] for an extensive discussion about the notion of $L^{1}$-dissipative germs and to [3] for a discussion on the correspondence between constraining the flux and choosing an undercompressive shock.

We focus now on the characterization of the relevant solution to (1). 
Definition 1.2 A function $u \in L^{\infty}\left(\mathbb{R} \times \mathbb{R}_{+} ;[0,1]\right)$ is said to be a solution of the problem (1) if:

1. for all $\kappa \in[0,1]$, for all $\psi \in \mathcal{D}^{+}\left(\mathbb{R} \times \mathbb{R}_{+}\right)^{1}$ such that $\psi(0, \cdot)=0$,

$$
\begin{aligned}
\int_{0}^{+\infty} \int_{\mathbb{R}}|u(x, t)-\kappa| \partial_{t} \psi(x, t) & d x d t+\int_{\mathbb{R}}\left|u_{0}(x)-\kappa\right| \psi(x, 0) d x \\
& +\int_{0}^{+\infty} \int_{\mathbb{R}} \Phi_{\kappa}(u)(x, t) \partial_{x} \psi(x, t) d x d t \geq 0 ;
\end{aligned}
$$

2. for almost every $t \in \mathbb{R}_{+}$, the one-sided traces $\left(u_{L}(t), u_{R}(t)\right)$ belong to $\mathcal{G}^{*}(t)$.

Remark 1.1 Three equivalent notions of solutions to the problem (1), among which the previous one, are proposed in [4]. Here, we choose to focus on only one of them, which is the only one that we will use in the sequel.

Let us describe now the $L^{1}$-dissipative germ $\mathcal{G}^{*}$ involved in the problem (1). Given a constraint $F \in$ $L^{\infty}\left(\mathbb{R}_{+},[0, f(\bar{u})]\right)$ and $A, B$ the functions defined by (9), then following [4], the $L^{1}$-dissipative germ $\mathcal{G}^{*}$ corresponding to the problem (1), represented in Figure 1, can be split into three parts

$$
\mathcal{G}^{*}(t)=\mathcal{G}_{1}(t) \cup \mathcal{G}_{2}(t) \cup \mathcal{G}_{3}(t),
$$

where

- $\mathcal{G}_{1}(t)=(A(t), B(t))$ corresponds to the unique undercompressive shock allowed at time $t>0$;

- $\mathcal{G}_{2}(t)=\left\{(c, c) \in[0,1]^{2} \mid f(c) \leq F(t)\right\}$ corresponds to a continuous solution across the interface and a flux satisfying the constraint;

- $\mathcal{G}_{3}(t)=\left\{\left(c_{L}, c_{R}\right) \in[0,1]^{2} \mid c_{L} \leq \bar{u}, c_{R} \geq \bar{u}\right.$ and $\left.f\left(c_{L}\right)=f\left(c_{R}\right) \leq F(t)\right\}$ correspond to the compressive stationary shocks satisfying the constraint.
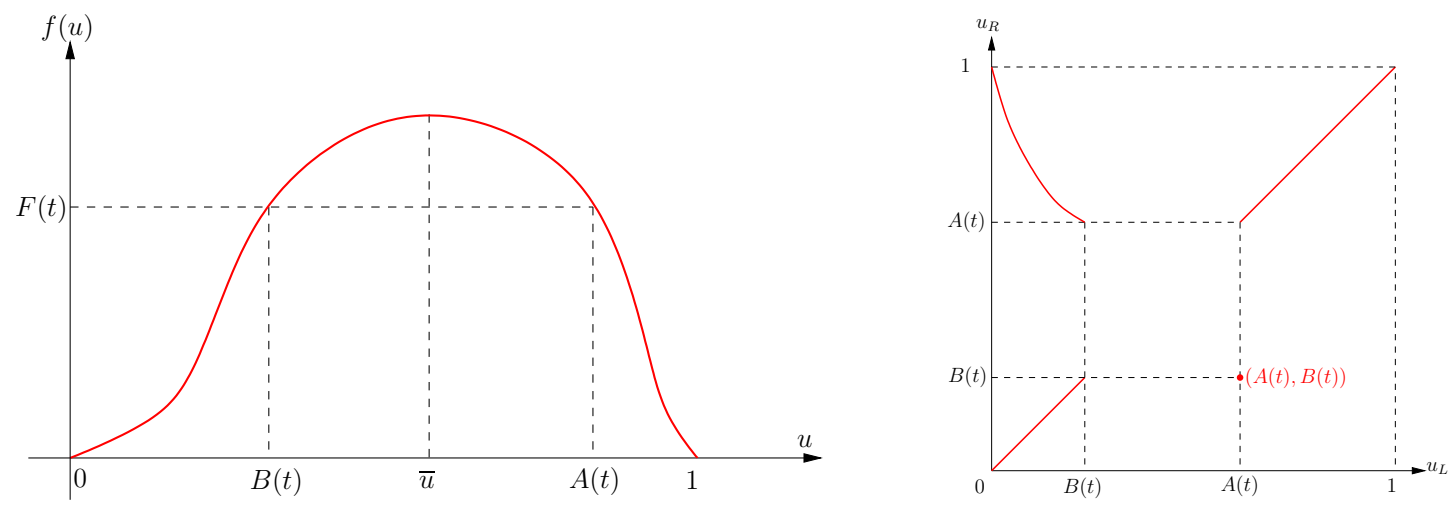

Figure 1: Left: graphical representation of the values $A(t)$ and $B(t)$ corresponding to the constraint $F(t)$. Right: graphical representation of the corresponding $L^{1}$-dissipative germ $\mathcal{G}^{*}(t)$.

We now state the existence and uniqueness result for the solution $u$ of the problem (1) whose proof is detailed in $[14,4]$. The time continuity of the solution $u$ prescribed below is a consequence of the result stated in [11].

Theorem $1([14,4])$ Let $u_{0} \in L^{\infty}(\mathbb{R} ;[0,1])$, and let $F \in L^{\infty}\left(\mathbb{R}_{+} ;[0, f(\bar{u})]\right)$, then there exists a unique $u$ solution to the problem (1) in the sense of the Definition 1.2, which furthermore can be assumed to belong to $\mathcal{C}\left(\mathbb{R}_{+}, L_{\text {loc }}^{1}(\mathbb{R})\right)$. Moreover, if $v$ is another solution corresponding to the initial data $v_{0} \in L^{\infty}(\mathbb{R} ;[0,1])$ such that $\left(u_{0}-v_{0}\right) \in L^{1}(\mathbb{R})$, then one has, for all $t \in \mathbb{R}_{+}$,

$$
\|u(\cdot, t)-v(\cdot, t)\|_{L^{1}(\mathbb{R})} \leq\left\|u_{0}-v_{0}\right\|_{L^{1}(\mathbb{R})} .
$$

\footnotetext{
${ }^{1}$ The set $\mathcal{D}^{+}\left(\mathbb{R} \times \mathbb{R}_{+}\right)$denotes the space of $\mathcal{C}^{\infty}\left(\mathbb{R} \times \mathbb{R}_{+} ; \mathbb{R}_{+}\right)$functions with compact support.
} 


\section{Godunov approximation of the problem and main result}

\subsection{The Godunov approximation}

In this section, we introduce the Godunov approximation of the problem (1). For the sake of simplicity, we choose to deal with uniform discretizations of $\mathbb{R} \times \mathbb{R}_{+}$. Nevertheless, note that all the following results can be adapted to the case of non-uniform discretizations.

Let $h>0$ the space step, we denote, for all $i \in \mathbb{Z}$,

$$
x_{i}=i h, \quad x_{i+1 / 2}=(i+1 / 2) h \quad \text { and } \quad u_{i+1 / 2}^{0}=\frac{1}{h} \int_{x_{i}}^{x_{i+1}} u_{0}(x) d x .
$$

Let $k>0$ the time step, we denote by $t^{n}=n k(n \in \mathbb{N})$, and by

$$
F^{n}=\frac{1}{k} \int_{t^{n}}^{t^{n+1}} F(t) d t, \quad A^{n}=\max \left\{s \in[0,1] \mid f(s)=F^{n}\right\}, \quad B^{n}=\min \left\{s \in[0,1] \mid f(s)=F^{n}\right\} .
$$

Note that $F^{n} \leq f(\bar{u})(n \in \mathbb{N})$.

We define the constrained Godunov scheme by

$$
\frac{u_{i+1 / 2}^{n+1}-u_{i+1 / 2}^{n}}{k} h+G_{i+1}^{n}\left(u_{i+1 / 2}^{n}, u_{i+3 / 2}^{n}\right)-G_{i}^{n}\left(u_{i-1 / 2}^{n}, u_{i+1 / 2}^{n}\right)=0,
$$

where $G_{i}^{n}$ is the Godunov numerical flux through the edge $x_{i}$, given by

- the classical Godunov numerical flux $G$ if $i \neq 0$, i.e.

$$
G_{i}^{n}(a, b)=G(a, b)=\min (f(\min \{a, \bar{u}\}), f(\max \{\bar{u}, b\}))= \begin{cases}\min _{s \in[a, b]} f(s) & \text { if } a \leq b, \\ \max _{s \in[b, a]} f(s) & \text { if } b \leq a\end{cases}
$$

- the constrained Godunov numerical flux $G_{0}^{n}$ if $i=0$, i.e.

$$
G_{0}^{n}(a, b)=\min \left(F^{n}, G(a, b)\right),
$$

as proposed in [4].

Proposition 2.1 The constrained Godunov numerical flux (14) coincides with the classical Godunov numerical flux for the constrained problem, i.e. $f(\mathcal{U}(0 ; a, b))$ where $\mathcal{U}(x / t ; a, b)$ is the self-similar solution of the constrained Riemann problem (described in [14]).

Proof: Consider $u_{0}(x)=a \mathbb{1}_{x<0}+b \mathbb{1}_{x>0}$. First, solving the Riemann problem without constraint, i.e.

$$
\begin{cases}\partial_{t} v+\partial_{x} f(v)=0 & \text { in } \mathbb{R} \times \mathbb{R}_{+}, \\ v_{\left.\right|_{t=0}}=a \mathbb{1}_{x<0}+b \mathbb{1}_{x>0} & \text { in } \mathbb{R}\end{cases}
$$

provides that either the solution $v$ is continuous at $x=0$ and $t>0$, with $v(0, t)=c$ that does not depend on $t$, or we have an compressive shock $f(a)=f(b)$ and $a<b$. In any case, the solution is self-similar, yielding that $t \mapsto f(v)(0, t)$ is constant on $(0,+\infty)$, and is exactly given by the formula (13).

Assume first that $f(v)(0, t) \leq F^{n}$, then, clearly, $v$ satisfies (10). Moreover, $\left(v\left(0^{-}, t\right), v\left(0^{+}, t\right)\right)$ either belongs to $\mathcal{G}_{2}(t)$ or $\mathcal{G}_{3}(t)$ (that do not depend on time, since we consider here the constraint $F \equiv F^{n}$ ). Therefore, $v$ is the unique solution of the problem (1) for the constant constraint $F \equiv F^{n}$.

Assume now that $f(v(0, t))>F^{n}$, then one deduces from the formula (13) and from the fact that $f$ is bell-shaped (2) that $a \geq B^{n}$ and $b \leq A^{n}$. In this case, define $u$ as the solution of two distinct initial boundary value problems

$$
\left\{\begin{array} { l l } 
{ \partial _ { t } u + \partial _ { x } f ( u ) = 0 } & { \text { in } \mathbb { R } _ { - } \times \mathbb { R } _ { + } } \\
{ u _ { | t = 0 } = a } & { \text { in } \mathbb { R } _ { - } } \\
{ u _ { | _ { x = 0 } } = A ^ { n } } & { \text { in } \mathbb { R } _ { + } , }
\end{array} \quad \left\{\begin{array}{ll}
\partial_{t} u+\partial_{x} f(u)=0 & \text { in } \mathbb{R}_{+} \times \mathbb{R}_{+} \\
u_{\mid t=0}=b & \text { in } \mathbb{R}_{+} \\
u_{\left.\right|_{x=0}}=B^{n} & \text { in } \mathbb{R}_{+} .
\end{array}\right.\right.
$$


Since $a \geq B^{n}$ (respectively $b \leq A^{n}$ ), the wave connecting $a$ to $A^{n}$ (resp. $B^{n}$ to $b$ ) has a non-positive (resp. non-negative) speed, so that the boundary condition is fulfilled in a strong sense in each case. Clearly, $u$ satisfies (10), and its traces on the interface belong to $\mathcal{G}_{3}(t)$, thus $u$ is the unique solution to the problem (1) for the constant constraint $F \equiv F^{n}$, and the flux at the interface is exactly given by $F^{n}$.

All along this paper, we assume that the following CFL condition is fulfilled:

$$
\frac{2 L_{f} k}{h} \leq 1
$$

and also that the time step is bounded, let say, without loss of generality,

$$
k \leq 1 \text {. }
$$

Definition 2.1 We denote by $u_{h}$ the so-called approximate solution, defined almost everywhere by

$$
u_{h}(x, t)=u_{i+1 / 2}^{n} \quad \text { if }(x, t) \in\left(x_{i}, x_{i+1}\right) \times\left[t^{n}, t^{n+1}\right) .
$$

We now state the $L^{\infty}$ stability of the scheme. We refer to [4, Proposition 4.2] for the proof of Proposition 2.2 .

Proposition 2.2 Under the CFL condition (15), then

$$
0 \leq u_{h} \leq 1 \quad \text { a.e. in } \mathbb{R} \times \mathbb{R}_{+} .
$$

\subsection{Approximate traces on the interface}

In this section, we seek to introduce, for all $n \in \mathbb{N}$, two artificial approximate traces $u_{L}^{n}, u_{R}^{n}$ such that $\left(u_{L}^{n}, u_{R}^{n}\right)$ belongs to the approximate germ $\mathcal{G}^{n}$, defined by

$$
\mathcal{G}^{n}=\mathcal{G}_{1}^{n} \cup \mathcal{G}_{2}^{n}
$$

with

$$
\mathcal{G}_{1}^{n}=\left(A^{n}, B^{n}\right), \quad \mathcal{G}_{2}^{n}=\left\{(c, c) \in[0,1]^{2} \mid f(c)<F^{n}\right\},
$$

and then to derive some properties on them.

Proposition 2.3 For all $n \in \mathbb{N}$, there exists $\left(u_{L}^{n}, u_{R}^{n}\right) \in \mathcal{G}^{n}$ such that

$$
G_{0}^{n}\left(u_{-1 / 2}^{n}, u_{1 / 2}^{n}\right)=G\left(u_{-1 / 2}^{n}, u_{L}^{n}\right)=G\left(u_{R}^{n}, u_{1 / 2}^{n}\right)=f\left(u_{L}^{n}\right)=f\left(u_{R}^{n}\right) .
$$

In the case where $G\left(u_{-1 / 2}^{n}, u_{1 / 2}^{n}\right)<F^{n}$, one has either $u_{L}^{n}=u_{R}^{n}=u_{-1 / 2}^{n}$ or $u_{L}^{n}=u_{R}^{n}=u_{1 / 2}^{n}$. Moreover, the case $u_{L}^{n}=A^{n}$ and $u_{R}^{n}=B^{n}$ only occurs when $u_{1 / 2}^{n} \leq A^{n}$ and $u_{-1 / 2}^{n} \geq B^{n}$.

Proof: We can prove the above Proposition by a case by case study. For reader's convenience, we drop the index $n$. Let $u_{-1 / 2}$ and $u_{1 / 2}$ belong to $[0,1]$, then we define by $u_{-1 / 2}^{\star} \in[\bar{u}, 1]$ and $u_{1 / 2}^{\star} \in[0, \bar{u}]$ by

$$
f\left(u_{-1 / 2}^{\star}\right)=f\left(u_{-1 / 2}\right), \quad f\left(u_{1 / 2}^{\star}\right)=f\left(u_{1 / 2}\right) .
$$

1. Assume that $0 \leq u_{-1 / 2}<B$.

(a) If $u_{1 / 2} \leq u_{-1 / 2}^{\star}$, then $u_{L}=u_{R}=u_{-1 / 2}$ satisfies (17).

(b) If $u_{1 / 2}>u_{-1 / 2}^{\star}$, then $u_{L}=u_{R}=u_{1 / 2}$ satisfies (17).

2. Assume that $B \leq u_{-1 / 2} \leq 1$.

(a) If $0 \leq u_{1 / 2} \leq A$, then $u_{L}=A$ and $u_{R}=B$ satisfies (17).

(b) If $A<u_{1 / 2} \leq 1$, then $u_{L}=u_{R}=u_{1 / 2}$ satisfies (17). 
Remark 2.1 1. Note that $u_{L}^{n}, u_{R}^{n}$ are not in general unique. Indeed, assume that $u_{-1 / 2}^{n}<B^{n}$, and that $u_{1 / 2}^{n}=u_{-1 / 2}^{n, \star}$, then $\left(u_{L}^{n}, u_{R}^{n}\right)$ can be chosen equal to either $\left(u_{-1 / 2}^{n}, u_{-1 / 2}^{n}\right)$ or $\left(u_{1 / 2}^{n}, u_{1 / 2}^{n}\right)$. Of course, whatever this choice is, the flux $f\left(u_{L}^{n}\right)=f\left(u_{R}^{n}\right)$ through the interface $\{x=0\}$ is unique.

2. The introduction of these approximate traces enables us to rewrite the constrained Godunov scheme (12)-(14) as two classical Godunov schemes on $\{x<0\}$ and $\{x>0\}$ with respective Dirichlet boundary conditions $u_{L}^{n}$ and $u_{R}^{n}$ :

$$
\begin{aligned}
& \frac{u_{i+1 / 2}^{n+1}-u_{i+1 / 2}^{n}}{k} h+G\left(u_{i+1 / 2}^{n}, u_{i+3 / 2}^{n}\right)-G\left(u_{i-1 / 2}^{n}, u_{i+1 / 2}^{n}\right)=0, \quad \text { for } i \notin\{-1,0\}, \\
& \frac{u_{-1 / 2}^{n+1}-u_{-1 / 2}^{n}}{k} h+G\left(u_{-1 / 2}^{n}, u_{L}^{n}\right)-G\left(u_{-3 / 2}^{n}, u_{-1 / 2}^{n}\right)=0, \\
& \frac{u_{1 / 2}^{n+1}-u_{1 / 2}^{n}}{k} h+G\left(u_{1 / 2}^{n}, u_{3 / 2}^{n}\right)-G\left(u_{R}^{n}, u_{1 / 2}^{n}\right)=0 .
\end{aligned}
$$

In the sequel, we denote by $u_{L, h}$ and $u_{R, h}$ the functions defined by

$$
u_{L, h}(t):=u_{L}^{n}, \quad u_{R, h}(t)=u_{R}^{n} \quad \text { for } t \in\left[t^{n}, t^{n+1}\right) .
$$

\subsection{Convergence of the scheme and error estimate}

We state here the following convergence result, which is the main result of [4].

Theorem $2([4])$ Let $F \in L^{\infty}\left(\mathbb{R}_{+} ;[0, f(\bar{u})]\right)$, and let $u_{0} \in L^{\infty}(\mathbb{R} ;[0,1])$, then, under the CFL condition

$$
\frac{2 L_{f} k}{h} \leq 1-\xi, \quad \text { with } \xi \in(0,1),
$$

then the approximate solution $u_{h}$ defined in Definition 2.1 converges in $L_{\text {loc }}^{1}\left(\mathbb{R} \times \mathbb{R}_{+}\right)$towards the unique solution $u$ to the problem in the sense of Definition 1.2 as $h$ tends to 0.

Remark 2.2 Note that the CFL condition (20) is slightly more restrictive than (15), so that some numerical diffusion stabilizes the scheme. However, we will deduce from this work that, if the data are sufficiently regular (roughly speaking in $\mathrm{BV}_{\text {loc }}$ ), then the scheme still converges under the weaker CFL condition (15).

Assumption 1 The functions $A$ and $B$ defined in (9) belong to $\mathrm{BV}_{\text {loc }}\left(\mathbb{R}_{+}\right)$.

In order to improve the error estimate, we shall make the following assumption.

Assumption 2 There exists $C_{\mathrm{BV}}$ depending only on $T$ such that, for all discretization parameters $h, k$ fulfilling (15), and for all $\tau \in(0, T)$,

$$
\int_{0}^{T-\tau}\left(\left|u_{L, h}(t+\tau)-u_{L, h}(t)\right|+\left|u_{R, h}(t+\tau)-u_{R, h}(t)\right|\right) d t \leq C_{\mathrm{BV}} .
$$

In the sequel, we denote by $\omega_{R}$ the subset of $\mathbb{R} \times \mathbb{R}_{+}$given by

$$
\omega_{R}=\left\{(x, t) \in \mathbb{R} \times \mathbb{R}_{+}|| x \mid \leq R-L_{f} t\right\} .
$$

Because the solution $u$ propagates with finite speed lower or equal to $L_{f}$, the restriction to $\omega_{R}$ of the solution $u$ depends only on the restriction of $u_{0}$ to $[-R, R]$.

Let us now state the main result of this paper. It relies on two error estimates between the approximate solution $u_{h}$ and the solution $u$. As usual, it is derived in the BV setting, but due to the (relatively slight) loss of control of the approximate traces $\left(u_{L}^{n}, u_{R}^{n}\right)_{n}$, the optimal order, that is $h^{1 / 2}$, is obtained under assumption 2 . 
Theorem 3 Let $u_{0} \in \mathrm{BV}_{\text {loc }}(\mathbb{R})$, let $u$ be the unique solution of the problem (1), and let $u_{h}$ be the approximate solution given by its Godunov approximation. Then, under the CFL condition (15) and Assumption 1, for all $R>0$, there exists $C$ depending only $u_{0}, f, A, B$ and $R$ such that

$$
\iint_{\omega_{R}}\left|u_{h}(x, t)-u(x, t)\right| d x d t \leq C h^{1 / 3} .
$$

Moreover, if Assumption 2 holds, then for all $R>0$, there exists $C$ depending only $u_{0}, f, A, B, C_{\mathrm{BV}}$ and $R$ such that

$$
\iint_{\omega_{R}}\left|u_{h}(x, t)-u(x, t)\right| d x d t \leq C h^{1 / 2} .
$$

Remark 2.3 In the particular case of a Riemann problem, then $u_{L, h}$ and $u_{R, h}$ are constant w.r.t. time, then Assumption 2 holds for $C_{B V}=0$, and the error estimate (23) holds.

The proof of Theorem 3 is based on the doubling variable technique introduced by S. N. KRUžKov [19] for proving the uniqueness of the solution of the entropy solution of a multidimensional scalar conservation law, and then used by N. N. Kuznetsov [20] in order to obtain some error estimate for the approximation of scalar conservation laws by monotone finite differences methods.

In order to obtain the convergence rates $1 / 3$ and $1 / 2$ stated in $(22)$ and (23), we need to show that

(i) the exact solution $u$ belongs to $\mathrm{BV}_{\text {loc }}\left(\mathbb{R} \times \mathbb{R}_{+}\right)$,

(ii) the approximate solution $u_{h}$ is uniformly bounded w.r.t. the discretization in $\mathrm{BV}_{\text {loc }}\left(\mathbb{R} \times \mathbb{R}_{+}\right)$.

\subsection{Outline of the paper}

We derive in Section 3 a uniform (w.r.t. the space step $h$ ) estimate for the local total variation of the approximate solution $u_{h}$. As a direct consequence, this will yield an estimate on the local total variation of the exact solution $u$. In section 4 , we provide the discrete and continuous entropy inequalities that the approximate solution verifies. Section 5 is devoted to the proof of Theorem 3, following N. N. KuZnETsov [20]. We illustrate our error estimates by numerical tests in Section 6 and discuss in Section 7 several perspectives which could follow this work.

\section{BV estimates}

We first derive BV estimates on the approximate solution. This requires a careful study of the approximate traces, based on an extended definition of the numerical total variation which incorporates the discrete traces. Secondly, we deduce BV estimates on the exact solution.

\subsection{BV estimate on the approximate constraint}

Lemma 3.1 Under Assumption 1, the functions $A_{h}, B_{h}$ defined respectively by $A_{h}(t)=A^{n}, B_{h}(t)=B^{n}$ if $t \in\left[t^{n}, t^{n+1}\right)$ belong to $\mathrm{BV}_{\text {loc }}\left(\mathbb{R}_{+}\right)$, and, for all $T>0$,

$$
T V_{[0, T]}\left(A_{h}-B_{h}\right)=T V_{[0, T]}\left(A_{h}\right)+T V_{[0, T]}\left(B_{h}\right) \leq T V_{[0, T]}(A)+T V_{[0, T]}(B)+1 .
$$

Proof: Assume first that the functions $A, B$ are smooth, so $F=f(A)=f(B)$ is Lipschitz continuous. Then, for all $n \in \mathbb{N}$, there exists $\tilde{t}^{n} \in\left(t^{n}, t^{n+1}\right)$ such that $F^{n}=F\left(\tilde{t}^{n}\right)$. Hence,

$$
A^{n}=A\left(\tilde{t}^{n}\right), \quad B^{n}=B\left(\tilde{t}^{n}\right) .
$$

Thus, denoting by $N$ the index of the time step where $t^{N} \leq T<t^{N+1}$, one gets that

$$
\begin{aligned}
T V_{[0, T]}\left(A_{h}\right) & =\sum_{n=0}^{N-1}\left|A^{n+1}-A^{n}\right|=\sum_{n=0}^{N-1}\left|A\left(\tilde{t}^{n+1}\right)-A\left(\tilde{t}^{n}\right)\right| \\
& =\sum_{n=0}^{N-2}\left|A\left(\tilde{t}^{n+1}\right)-A\left(\tilde{t}^{n}\right)\right|+\left|A\left(\tilde{t}^{N}\right)-A\left(\tilde{t}^{N-1}\right)\right| .
\end{aligned}
$$


Since

$$
\left|A\left(\tilde{t}^{N}\right)-A\left(\tilde{t}^{N-1}\right)\right| \leq 1-\bar{u}
$$

and

one obtains that

$$
\sum_{n=0}^{N-2}\left|A\left(\tilde{t}^{n+1}\right)-A\left(\tilde{t}^{n}\right)\right| \leq T V_{[0, T]}(A),
$$

Similarly, we can state that

$$
T V_{[0, T]}\left(A_{h}\right) \leq T V_{[0, T]}(A)+(1-\bar{u}) .
$$

$$
T V_{[0, T]}\left(B_{h}\right) \leq T V_{[0, T]}(B)+\bar{u}
$$

so that the estimate (24) holds for smooth functions $A, B$. Assume now that $A, B$ only belong to $\mathrm{BV}_{\text {loc }}\left(\mathbb{R}_{+}\right)$, then there exists some sequences $\left(A_{\nu}\right)_{\nu \in \mathbb{N}}$ and $\left(B_{\nu}\right)_{\nu \in \mathbb{N}}$ of smooth functions (obtained for example by convolution with smoothing kernels) such that

$$
\begin{gathered}
A_{\nu} \rightarrow A, \quad B_{\nu} \rightarrow B \quad \text { a.e. in } \mathbb{R}_{+} \text {as } \nu \rightarrow \infty, \\
T V_{[0, T]}\left(A_{\nu}\right) \leq T V_{[0, T]}(A), \quad T V_{[0, T]}\left(B_{\nu}\right) \leq T V_{[0, T]}(B),
\end{gathered}
$$

and

$$
A_{\nu, h} \rightarrow A_{h}, \quad B_{\nu, h} \rightarrow B_{h} \text { a.e. in } \mathbb{R}_{+} \text {as } \nu \rightarrow \infty .
$$

Then we can pass to the limit and extend (24) to functions $A, B$ merely in $\mathrm{BV}_{\text {loc }}\left(\mathbb{R}_{+}\right)$.

It only remains to check that due to their definition (11) and to the bell-shaped behavior of the function $f$, the functions $n \rightarrow A^{n}$ and $n \rightarrow B^{n}$ have variations of opposite signs, i.e.

$$
A^{n+1} \geq A^{n} \quad \Leftrightarrow \quad F^{n+1} \leq F^{n} \quad \Leftrightarrow \quad B^{n+1} \leq B^{n},
$$

yielding $T V\left(A_{h}-B_{h}\right)=T V\left(A_{h}\right)+T V\left(B_{h}\right)$.

\subsection{Space BV estimate on the approximate solution}

In the sequel, we introduce a modified total variation, that takes the approximate traces into account:

$$
\mathcal{T V}\left(u_{h}\left(\cdot, t^{n}\right)\right)=\sum_{i \neq 0}\left|u_{i+1 / 2}^{n}-u_{i-1 / 2}^{n}\right|+\left|u_{-1 / 2}^{n}-u_{L}^{n}\right|+\left|u_{1 / 2}^{n}-u_{R}^{n}\right|
$$

while the classical total variation of $u_{h}\left(\cdot, t^{n}\right)$ is given by

$$
T V\left(u_{h}\left(\cdot, t^{n}\right)\right)=\sum_{i \in \mathbb{Z}}\left|u_{i+1 / 2}^{n}-u_{i-1 / 2}^{n}\right| \cdot
$$

We first state the following technical lemma.

Lemma 3.2 Let $(c, d) \in[0,1]^{2}$ such that $c \geq d$. Then, for all $(a, b) \in[d, 1] \times[0, c]$, one has

$$
|| c-a|+| d-b|-| a-b|| \leq c-d .
$$

Proof: Denote by $\Psi(a, b)=|c-a|+|d-b|-|a-b|$. The proof is performed using a case by case study, summarized in the following table. Note that only 4 cases are used in the proof of Lemma 3.2, but the other cases will be used later. In this table, we denote by $a \top b=\max (a, b)$ and $a \perp b=\min (a, b)$.

\begin{tabular}{|c|c|c|c|}
\hline & $a \in[0, d]$ & $a \in[d, c]$ & $a \in[c, 1]$ \\
\hline$b \in[0, d]$ & $\Psi(a, b)=c+d-2(a \top b)$ & $\Psi(a, b)=c+d-2 a$ & $\Psi(a, b)=d-c$ \\
\hline$b \in[d, c]$ & $\Psi(a, b)=c-d$ & $\Psi(a, b)=c-d-2(a-b)^{+}$ & $\Psi(a, b)=2 b-(d+c)$ \\
\hline$b \in[c, 1]$ & $\Psi(a, b)=c-d$ & $\Psi(a, b)=c-d$ & $\Psi(a, b)=2(a \perp b)-(c+d)$ \\
\hline
\end{tabular}


Lemma 3.3 For all $t \geq 0$, one has

$$
\left|\mathcal{T} \mathcal{V}\left(u_{h}(\cdot, t)\right)-T V\left(u_{h}(\cdot, t)\right)\right| \leq 1
$$

Proof: Let $t \in\left[t^{n}, t^{n+1}\right)$. Assume that $\left(u_{L}^{n}, u_{R}^{n}\right) \in \mathcal{G}_{2}^{n}$, then $\mathcal{T} \mathcal{V}\left(u_{h}(\cdot, t)\right)=T V\left(u_{h}(\cdot, t)\right)$. We now focus on the case where $\left(u_{L}^{n}, u_{R}^{n}\right)=\left(A^{n}, B^{n}\right)$. As seen in Proposition 2.3, this implies that $u_{-1 / 2}^{n} \geq B^{n}$ and $u_{1 / 2}^{n} \leq A^{n}$. Since

$$
\mathcal{T V}\left(u_{h}(\cdot, t)\right)-T V\left(u_{h}(\cdot, t)\right)=\left|u_{-1 / 2}^{n}-A^{n}\right|+\left|u_{1 / 2}^{n}-B^{n}\right|-\left|u_{-1 / 2}^{n}-u_{1 / 2}^{n}\right|,
$$

then using the lemma 3.2 with $a=u_{-1 / 2}^{n}, b=u_{1 / 2}^{n}, c=A^{n}, d=B^{n}$ provides the result.

Denote by $\Lambda \subset \mathbb{N}$ the set of the time step indices where the constraint is saturated, i.e.

$$
p \in \Lambda \quad \Leftrightarrow \quad u_{-1 / 2}^{p} \geq B^{p} \text { and } u_{1 / 2}^{p} \leq A^{p} \quad\left(\Leftrightarrow \quad G\left(u_{-1 / 2}^{p}, u_{1 / 2}^{p}\right) \geq F^{p}\right),
$$

by

$$
\underline{\Lambda}=\{p \in \mathbb{N} \mid p \notin \Lambda, p+1 \in \Lambda\}, \quad \bar{\Lambda}=\{p \in \Lambda \mid p+1 \notin \Lambda\}, \quad \stackrel{\circ}{\Lambda}=\Lambda \backslash \bar{\Lambda},
$$

and by

$$
\Upsilon=\mathbb{N} \backslash(\underline{\Lambda} \cup \Lambda)=\{p \in \mathbb{N} \mid p \notin \Lambda,(p+1) \notin \Lambda\},
$$

so that we have

$$
\mathbb{N}=\Upsilon \cup \stackrel{\circ}{\cup} \underline{\Lambda} \cup \bar{\Lambda}
$$

Lemma 3.4 Let $p \in \Upsilon$, then, under the CFL condition (15),

$$
\mathcal{T} \mathcal{V}\left(u_{h}\left(\cdot, t^{p+1}\right)\right) \leq \mathcal{T} \mathcal{V}\left(u_{h}\left(\cdot, t^{p}\right)\right) .
$$

Proof: Since $p \notin \Lambda$, then $u_{h}\left(\cdot, t^{p+1}\right)$ is the solution computed by the classical Godunov scheme without constraint. Hence, it follows from classical computations (see e.g. [17] or Lemma 5.7 in [16]) that

$$
\sum_{i \in \mathbb{Z}}\left|u_{i+1 / 2}^{p+1}-u_{i-1 / 2}^{p+1}\right| \leq \sum_{i \in \mathbb{Z}}\left|u_{i+1 / 2}^{p}-u_{i-1 / 2}^{p}\right| .
$$

Now, since $p \in \Upsilon$ then, thanks to Proposition 2.3, for $q \in\{p, p+1\}$, either $u_{L}^{q}=u_{R}^{q}=u_{-1 / 2}^{q}$ or $u_{L}^{q}=u_{R}^{q}=$ $u_{1 / 2}^{q}$. As a consequence,

$$
\mathcal{T} \mathcal{V}\left(u_{h}\left(\cdot, t^{q}\right)\right)=\sum_{i \in \mathbb{Z}}\left|u_{i+1 / 2}^{q}-u_{i-1 / 2}^{q}\right| .
$$

Lemma 3.4 is a direct consequence of (26) and (27).

Lemma 3.5 Let $p \in \underline{\Lambda}$, then, under the CFL condition (15),

$$
\mathcal{T} \mathcal{V}\left(u_{h}\left(\cdot, t^{p+1}\right)\right) \leq \mathcal{T} \mathcal{V}\left(u_{h}\left(\cdot, t^{p}\right)\right)+\left(A^{p+1}-B^{p+1}\right) .
$$

Proof: Since $p \notin \Lambda$, then, as previously,

$$
\sum_{i \in \mathbb{Z}}\left|u_{i+1 / 2}^{p+1}-u_{i-1 / 2}^{p+1}\right| \leq \sum_{i \in \mathbb{Z}}\left|u_{i+1 / 2}^{p}-u_{i-1 / 2}^{p}\right|=\mathcal{T} \mathcal{V}\left(u_{h}\left(\cdot, t^{p}\right)\right) .
$$

Since $\left(u_{L}^{p+1}, u_{R}^{p+1}\right)=\left(A^{p+1}, B^{p+1}\right)$, then

$$
\mathcal{T} \mathcal{V}\left(u_{h}\left(\cdot, t^{p+1}\right)\right) \leq \mathcal{T} \mathcal{V}\left(u_{h}\left(\cdot, t^{p}\right)\right)+\mathcal{R}^{p+1},
$$

where

$$
\mathcal{R}^{p+1}=\left|u_{-1 / 2}^{p+1}-A^{p+1}\right|+\left|u_{1 / 2}^{p+1}-B^{p+1}\right|-\left|u_{1 / 2}^{p+1}-u_{-1 / 2}^{p+1}\right| .
$$

Since $p+1 \in \Lambda$, then one has, thanks to Proposition 2.3,

$$
u_{-1 / 2}^{p+1} \geq B^{p+1} \quad \text { and } \quad u_{1 / 2}^{p+1} \leq A^{p+1} .
$$


Hence we can apply Lemma 3.2 to claim that

$$
\mathcal{R}^{p+1} \leq A^{p+1}-B^{p+1} .
$$

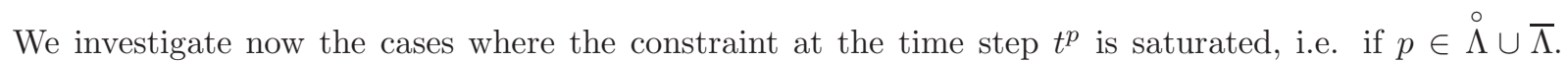
In these cases, $\left(u_{L}^{p}, u_{R}^{p}\right)=\left(A^{p}, B^{p}\right)$, and adapting once again the computations of [16, Lemma 5.7] on the formulation (18), we get that, under the CFL condition (15),

$$
\begin{aligned}
\sum_{i>0}\left|u_{i+1 / 2}^{p+1}-u_{i-1 / 2}^{p+1}\right|+\left|u_{1 / 2}^{p+1}-B^{p}\right| & \leq \sum_{i>0}\left|u_{i+1 / 2}^{p}-u_{i-1 / 2}^{p}\right|+\left|u_{1 / 2}^{p}-B^{p}\right| . \\
\sum_{i<0}\left|u_{i+1 / 2}^{p+1}-u_{i-1 / 2}^{p+1}\right|+\left|u_{-1 / 2}^{p+1}-A^{p}\right| & \leq \sum_{i<0}\left|u_{i+1 / 2}^{p}-u_{i-1 / 2}^{p}\right|+\left|u_{-1 / 2}^{p}-A^{p}\right| .
\end{aligned}
$$

As a direct consequence of the inequalities stated above, one has

$$
\mathcal{T} \mathcal{V}\left(u_{h}\left(\cdot, t^{p+1}\right)\right) \leq \mathcal{T} \mathcal{V}\left(u_{h}\left(\cdot, t^{p}\right)\right)+\mathcal{R}^{p+1},
$$

where

$$
\mathcal{R}^{p+1}=\left|u_{-1 / 2}^{p+1}-u_{L}^{p+1}\right|-\left|u_{-1 / 2}^{p+1}-A^{p}\right|+\left|u_{1 / 2}^{p+1}-u_{R}^{p+1}\right|-\left|u_{1 / 2}^{p+1}-B^{p}\right| .
$$

Lemma 3.6 Let $p \in \stackrel{\circ}{\Lambda}$, then, under the CFL condition (15),

$$
\mathcal{T} \mathcal{V}\left(u_{h}\left(\cdot, t^{p+1}\right)\right) \leq \mathcal{T} \mathcal{V}\left(u_{h}\left(\cdot, t^{p}\right)\right)+\left|\left(A^{p+1}-B^{p+1}\right)-\left(A^{p}-B^{p}\right)\right| .
$$

Proof: Since $p+1 \in \Lambda$, one has $\left(u_{L}^{p+1}, u_{R}^{p+1}\right)=\left(A^{p+1}, B^{p+1}\right)$. Replacing in (31) leads to, thanks to the triangle inequality,

$$
\mathcal{R}^{p+1} \leq\left|A^{p+1}-A^{p}\right|+\left|B^{p+1}-B^{p}\right|
$$

Since

$$
A^{p+1} \geq A^{p} \Leftrightarrow B^{p+1} \leq B^{p},
$$

one obtains that

$$
\mathcal{R}^{p+1} \leq\left|A^{p+1}-A^{p}\right|+\left|B^{p+1}-B^{p}\right|=\left|\left(A^{p+1}-B^{p+1}\right)-\left(A^{p}-B^{p}\right)\right| .
$$

We conclude by using (32) in (30).

Lemma 3.7 Let $p \in \bar{\Lambda}$, then, under the CFL condition (15),

$$
\mathcal{T} \mathcal{V}\left(u_{h}\left(\cdot, t^{p+1}\right)\right) \leq \mathcal{T} \mathcal{V}\left(u_{h}\left(\cdot, t^{p}\right)\right)+\left(B^{p+1}-A^{p+1}\right)+\left|A^{p+1}-A^{p}\right|+\left|B^{p+1}-B^{p}\right| .
$$

Proof: Since, thanks to Proposition 2.3, $u_{L}^{p+1}=u_{R}^{p+1} \in\left\{u_{-1 / 2}^{p+1}, u_{1 / 2}^{p+1}\right\}$, then the expression (31) turns to

$$
\mathcal{R}^{p+1} \leq \mathcal{R}_{1}^{p+1}+\left|A^{p+1}-A^{p}\right|+\left|B^{p+1}-B^{p}\right|,
$$

with

$$
\mathcal{R}_{1}^{p+1}=\left|u_{1 / 2}^{p+1}-u_{-1 / 2}^{p+1}\right|-\left|u_{-1 / 2}^{p+1}-A^{p+1}\right|-\left|u_{1 / 2}^{p+1}-B^{p+1}\right|
$$

Since $p+1 \notin \Lambda$, we known from the case by case study carried out in Proposition 2.3 that $u_{-1 / 2}^{p+1} \leq B^{p+1}$ or $u_{1 / 2}^{p+1} \geq A^{p+1}$. We deduce from the first column and the last line of (25) that $\mathcal{R}_{1}^{p+1} \leq B^{p+1}-A^{p+1}$.

Lemma 3.8 Assume that $u_{0} \in \mathrm{BV}(\mathbb{R})$, then, under the CFL condition (15) and Assumption 1, there exists $C$ depending only on $A, B$ and $T$ (but neither on $h$ nor on $k$ ) such that

$$
T V\left(u_{h}(\cdot, T)\right) \leq T V\left(u_{0}\right)+C .
$$


Proof: $\quad$ Let $n \in \mathbb{N}$ be such that $T \in\left[t^{n}, t^{n+1}\right)$, then

$$
\mathcal{T} \mathcal{V}\left(u_{h}(\cdot, T)\right)=\mathcal{T} \mathcal{V}\left(u_{h}(\cdot, 0)\right)+\sum_{p=0}^{n-1}\left(\mathcal{T} \mathcal{V}\left(u_{h}\left(\cdot, t^{p+1}\right)\right)-\mathcal{T} \mathcal{V}\left(u_{h}\left(\cdot, t^{p}\right)\right)\right)
$$

From Lemmata 3.4, 3.5, 3.6 and 3.7, we deduce that

$$
\mathcal{T V}\left(u_{h}\left(\cdot, t^{p+1}\right)\right)-\mathcal{T} \mathcal{V}\left(u_{h}\left(\cdot, t^{p}\right)\right) \leq \begin{cases}0, & \text { if } p \in \Upsilon, \\ A^{p+1}-B^{p+1}, & \text { if } p \in \underline{\Lambda}, \\ \left|\left(A^{p+1}-B^{p+1}\right)-\left(A^{p}-B^{p}\right)\right|, & \text { if } p \in \Lambda, \\ B^{p+1}-A^{p+1}+\left|\left(A^{p+1}-B^{p+1}\right)-\left(A^{p}-B^{p}\right)\right| & \text { if } p \in \bar{\Lambda} .\end{cases}
$$

Therefore,

$$
\begin{aligned}
\mathcal{T V}\left(u_{h}(\cdot, T)\right) \leq & \mathcal{T} \mathcal{V}\left(u_{h}(\cdot, 0)\right)+\sum_{\substack{p \leq n-1 \\
p \in \Lambda}}\left|\left(A^{p+1}-B^{p+1}\right)-\left(A^{p}-B^{p}\right)\right| \\
& +\sum_{\substack{p \leq n-1 \\
p \in \underline{\Lambda}}}\left(A^{p+1}-B^{p+1}\right)+\sum_{\substack{p \leq n-1 \\
p \in \bar{\Lambda}}}\left(B^{p+1}-A^{p+1}\right) .
\end{aligned}
$$

Since for all $p, q \in \underline{\Lambda}$ with $p<q$, there exists $r \in \bar{\Lambda}$ such that $p<r<q$, and since $\left|A^{k}-B^{k}\right| \leq 1$ for all $k$, it follows that

$$
\sum_{\substack{p \leq n-1 \\ p \in \Lambda}}\left(\left(A^{p}-B^{p}\right)-\left(A^{p+1}-B^{p+1}\right)\right) \geq\left(A^{0}-B^{0}\right) \mathbb{1}_{\Lambda}(0)+\sum_{\substack{p \leq n-1 \\ p \in \underline{\Lambda}}}\left(A^{p+1}-B^{p+1}\right)+\sum_{\substack{p \leq n-1 \\ p \in \bar{\Lambda}}}\left(B^{p+1}-A^{p+1}\right)-1
$$

where the last term -1 is a lower bound to $A^{n}-B^{n}$. This inequality can also be written as

$$
\sum_{\substack{p \leq n-1 \\ p \in \underline{\Lambda}}}\left(A^{p+1}-B^{p+1}\right)+\sum_{\substack{p \leq n-1 \\ p \in \bar{\Lambda}}}\left(B^{p+1}-A^{p+1}\right) \leq 1+\sum_{\substack{p \leq n-1 \\ p \in \Lambda}}\left|\left(A^{p+1}-B^{p+1}\right)-\left(A^{p}-B^{p}\right)\right|
$$

which, taking (35) into account in (34), yields

$$
\begin{aligned}
\mathcal{T V}\left(u_{h}(\cdot, T)\right) & \leq \mathcal{T V}\left(u_{h}(\cdot, 0)\right)+1+2 \sum_{\substack{p \leq n-1 \\
p \in \Lambda}}\left|\left(A^{p+1}-B^{p+1}\right)-\left(A^{p}-B^{p}\right)\right| \\
& \leq \mathcal{T V}\left(u_{h}(\cdot, 0)\right)+1+2 T V_{[0, T]}\left(A_{h}-B_{h}\right) .
\end{aligned}
$$

We conclude by using Lemmata 3.1 and 3.3.

Proposition 3.9 Let $u_{0} \in \mathrm{BV}(\mathbb{R})$, then, under the CFL condition (15) and Assumption 1, there exists $C$ depending only on $u_{0}, T, A, B$ such that, for all $\xi>0$,

$$
\int_{0}^{T} \int_{\mathbb{R}}\left|u_{h}(x+\xi, t)-u_{h}(x, t)\right| d x d t \leq C \xi .
$$

Proof: It follows from Lemma 3.8 that the function $u_{h}(\cdot, t)$ has a bounded variation for all $t \in[0, T]$, thus there exists $C$ depending only on $u_{0}, A, B, T$ such that

$$
\int_{\mathbb{R}}\left|u_{h}(x+\xi, t)-u_{h}(x, t)\right| d x \leq C \xi .
$$

We conclude by integrating w.r.t. to $t \in[0, T]$. 


\subsection{Time BV estimate on the approximate solution}

Lemma 3.10 Let $u_{0} \in \mathrm{BV}(\mathbb{R})$, then, one has

$$
\sum_{i \in \mathbb{Z}}\left|u_{i+1 / 2}^{n+1}-u_{i+1 / 2}^{n}\right| \leq \frac{2 L_{f} k}{h} \mathcal{T} \mathcal{V}\left(u_{h}\left(\cdot, t^{n}\right)\right) .
$$

Proof: The scheme (12) can be rewritten

$$
\begin{aligned}
u_{i+1 / 2}^{n+1}-u_{i+1 / 2}^{n} & =\frac{k}{h}\left(G\left(u_{i-1 / 2}^{n}, u_{i+1 / 2}^{n}\right)-f\left(u_{i+1 / 2}^{n}\right)-\left(G\left(u_{i+1 / 2}^{n}, u_{i+3 / 2}^{n}\right)-f\left(u_{i+1 / 2}^{n}\right)\right)\right) \text { if } i \notin\{-1,0\} \\
u_{-1 / 2}^{n+1}-u_{-1 / 2}^{n} & =\frac{k}{h}\left(G\left(u_{-3 / 2}^{n}, u_{-1 / 2}^{n}\right)-f\left(u_{-1 / 2}^{n}\right)-\left(G\left(u_{-1 / 2}^{n}, u_{L}^{n}\right)-f\left(u_{-1 / 2}^{n}\right)\right)\right) \\
u_{1 / 2}^{n+1}-u_{1 / 2}^{n} & =\frac{k}{h}\left(G\left(u_{R}^{n}, u_{1 / 2}^{n}\right)-f\left(u_{1 / 2}^{n}\right)-\left(G\left(u_{1 / 2}^{n}, u_{3 / 2}^{n}\right)-f\left(u_{1 / 2}^{n}\right)\right)\right) .
\end{aligned}
$$

Using the fact that $G$ is $L_{f}$-Lipschitz continuous w.r.t. each of its variables, we obtain that

$$
\begin{aligned}
\left|u_{i+1 / 2}^{n+1}-u_{i+1 / 2}^{n}\right| & \leq \frac{L_{f} k}{h}\left(\left|u_{i-1 / 2}^{n}-u_{i+1 / 2}^{n}\right|+\left|u_{i+1 / 2}^{n}-u_{i+3 / 2}^{n}\right|\right) \quad \text { if } i \notin\{-1,0\}, \\
\left|u_{-1 / 2}^{n+1}-u_{-1 / 2}^{n}\right| & \leq \frac{L_{f} k}{h}\left(\left|u_{L}^{n}-u_{-1 / 2}^{n}\right|+\left|u_{-1 / 2}^{n}-u_{-3 / 2}^{n}\right|\right), \\
\left|u_{1 / 2}^{n+1}-u_{1 / 2}^{n}\right| & \leq \frac{L_{f} k}{h}\left(\left|u_{1 / 2}^{n}-u_{R}^{n}\right|+\left|u_{3 / 2}^{n}-u_{1 / 2}^{n}\right|\right) .
\end{aligned}
$$

Summing (37) for $i \in \mathbb{Z} \backslash\{-1,0\}$ with (38) and (39) yields (36).

Proposition 3.11 Let $u_{0} \in \mathrm{BV}(\mathbb{R})$, then, under the CFL condition (15), (16) and Assumption 1, for all $T>0$, there exists $C$ depending only on $A, B, T, u_{0}$ and $L_{f}$ such that, for all $\tau \in(0, T)$,

$$
\int_{0}^{T-\tau} \int_{\mathbb{R}}\left|u_{h}(x, t+\tau)-u_{h}(x, t)\right| d x d t \leq C \tau .
$$

Proof: One has (with a slight abuse of notation, since $\partial_{t} u_{h}$ is a bounded Radon measure on $\mathbb{R} \times[0, T]$ which is not absolutely continuous w.r.t. to the Lebesgue measure)

$$
\int_{\mathbb{R}} \int_{0}^{T}\left|\partial_{t} u_{h}(x, t)\right| d x d t=\sum_{i \in \mathbb{Z}} \sum_{n=0}^{\lfloor T / k\rfloor}\left|u_{i+1 / 2}^{n+1}-u_{i+1 / 2}^{n}\right| h .
$$

Then it follows from Lemmata 3.10 and 3.8 that

$$
\int_{\mathbb{R}} \int_{0}^{T}\left|\partial_{t} u_{h}(x, t)\right| d x d t \leq C \sum_{n=0}^{\lfloor T / k\rfloor} k
$$

Using (16), we obtain that

$$
\int_{\mathbb{R}} \int_{0}^{T}\left|\partial_{t} u_{h}(x, t)\right| d x d t \leq C .
$$

The inequality (40) is a classical consequence of the previous estimate (see e.g. [6]).

\section{4 $B V$ estimates on the exact solution}

Letting now $h$ tend to 0 , since we know, thanks to Theorem 2 , that $u_{h}$ tends to the unique solution $u$ (at least under the more restrictive CFL condition (20)) we obtain the following regularity result on the exact solution $u$.

Proposition 3.12 Let $u$ be the exact solution to the problem corresponding to $u_{0} \in \mathrm{BV}(\mathbb{R})$. Then, under Assumption 1, then, for all $T>0, u \in \mathrm{BV}(\mathbb{R} \times[0, T])$.

Remark 3.1 Because of the finite speed propagation property, the solution $u$ to the problem depends, on the set $\omega_{R}$ defined in (21), only on the restriction of the initial data $u_{0}$ to $[-R, R]$. So, if $u_{0} \in \mathrm{BV}_{\text {loc }}(\mathbb{R})$, extending $u_{0}$ by a constant outside of $[-R, R]$ will not affect the solution $u$ on $\omega_{R}$. Thus the Proposition 3.12 can be generalized in the following way. If $u_{0} \in \mathrm{BV}_{\text {loc }}(\mathbb{R})$, then, under Assumption 1 , u belongs to $\mathrm{BV}_{\text {loc }}\left(\mathbb{R} \times \mathbb{R}_{+}\right)$. 


\section{Entropy formulations for the approximate solution}

\subsection{Discrete entropy inequalities}

Using the approximate traces $u_{L}^{n}, u_{R}^{n}$ introduced in Section 2.2, the scheme (18) can be rewritten under the form

$$
\begin{gathered}
H\left(u_{i+1 / 2}^{n+1}, u_{i+1 / 2}^{n}, u_{i-1 / 2}^{n}, u_{i+3 / 2}^{n}\right)=0, \quad \forall i \notin\{-1,0\}, \\
H\left(u_{-1 / 2}^{n+1}, u_{-1 / 2}^{n}, u_{-3 / 2}^{n}, u_{L}^{n}\right)=0 \\
H\left(u_{1 / 2}^{n+1}, u_{1 / 2}^{n}, u_{R}^{n}, u_{3 / 2}^{n}\right)=0
\end{gathered}
$$

where, under the CFL condition (15), the function $H$ is non-decreasing w.r.t. its first argument, and nonincreasing w.r.t. its three last arguments. As a consequence, if $(a, b, c, d) \in[0,1]^{4}$ satisfies

$$
H(a, b, c, d)=0,
$$

then, thanks to the fact that, for all $\kappa \in[0,1]$,

$$
H(\kappa, \kappa, \kappa, \kappa)=0,
$$

it follows from classical computations (see e.g. [16]) that

$$
H(a \top \kappa, b \top \kappa, c \top \kappa, d \top \kappa)-H(a \perp \kappa, b \perp \kappa, c \perp \kappa, d \perp \kappa) \leq 0,
$$

where $a \top \kappa=\max (a, \kappa)$ and $a \perp \kappa=\min (a, \kappa)$. In the sequel, we denote by

$$
\Phi_{\kappa}(a, b)=G(a \top \kappa, b \top \kappa)-G(a \perp \kappa, b \perp \kappa) .
$$

Note that for all $a \in[0,1]$, for all $\kappa \in[0,1], \Phi_{\kappa}(a, a)=\Phi_{\kappa}(a)$, where the notation $\Phi_{\kappa}(a)$ has been introduced in (5). The following proposition follows from (42).

Proposition 4.1 For all $\kappa \in[0,1]$, one has

$$
\begin{aligned}
& \frac{\left|u_{i+1 / 2}^{n+1}-\kappa\right|-\left|u_{i+1 / 2}^{n}-\kappa\right|}{k} h+\Phi_{\kappa}\left(u_{i+1 / 2}^{n}, u_{i+3 / 2}^{n}\right)-\Phi_{\kappa}\left(u_{i-1 / 2}^{n}, u_{i+1 / 2}^{n}\right) \leq 0, \quad \forall i \in \mathbb{Z} \backslash\{-1,0\}, \\
& \frac{\left|u_{-1 / 2}^{n+1}-\kappa\right|-\left|u_{-1 / 2}^{n}-\kappa\right|}{k} h+\Phi_{\kappa}\left(u_{-1 / 2}^{n}, u_{L}^{n}\right)-\Phi_{\kappa}\left(u_{-3 / 2}^{n}, u_{-1 / 2}^{n}\right) \leq 0, \\
& \frac{\left|u_{1 / 2}^{n+1}-\kappa\right|-\left|u_{1 / 2}^{n}-\kappa\right|}{k} h+\Phi_{\kappa}\left(u_{1 / 2}^{n}, u_{3 / 2}^{n}\right)-\Phi_{\kappa}\left(u_{R}^{n}, u_{1 / 2}^{n}\right) \leq 0 .
\end{aligned}
$$

Lemma 4.2 For all $\kappa \in[0,1]$,

$$
\begin{gathered}
\Phi_{\kappa}\left(u_{-1 / 2}^{n}, u_{L}^{n}\right)-\Phi_{\kappa}\left(u_{L}^{n}\right) \geq 0, \\
\Phi_{\kappa}\left(u_{R}^{n}\right)-\Phi_{\kappa}\left(u_{R}^{n}, u_{1 / 2}^{n}\right) \geq 0 .
\end{gathered}
$$

Proof: We only prove (46), since the proof of (47) is similar. Here again, for readers convenience, we drop the index $n$. We denote by $I(a, b)$ the interval $[a, b]$ if $a \leq b$ and $[b, a]$ otherwise.

Firstly, if $\kappa \notin I\left(u_{-1 / 2}, u_{L}\right)$, then, using that $G\left(u_{-1 / 2}, u_{L}\right)=f\left(u_{L}\right)$, one has

$$
\Phi_{\kappa}\left(u_{-1 / 2}, u_{L}\right)=\Phi_{\kappa}\left(u_{L}\right) .
$$

Consider now the case where $\kappa \in I\left(u_{-1 / 2}, u_{L}\right)$. Since $G\left(u_{-1 / 2}, u_{L}\right)=f\left(u_{L}\right)$, the function $a \mapsto G\left(a, u_{L}\right)$ is constant on $I\left(u_{-1 / 2}, u_{L}\right)$. Assume that $u_{-1 / 2} \geq u_{L}$, then

$$
\Phi_{\kappa}\left(u_{-1 / 2}, u_{L}\right)=G\left(u_{-1 / 2}, \kappa\right)-G\left(\kappa, u_{L}\right)=G\left(u_{-1 / 2}, \kappa\right)-f\left(u_{L}\right) .
$$

Since $G$ is non-increasing w.r.t. its second argument, $G\left(u_{-1 / 2}, \kappa\right) \geq f(\kappa)$, hence one has

$$
\Phi_{\kappa}\left(u_{-1 / 2}, u_{L}\right) \geq f(\kappa)-f\left(u_{L}\right)=\Phi_{\kappa}\left(u_{L}\right) .
$$

Similarly, if $u_{-1 / 2} \leq u_{L}$, one obtains $\Phi_{\kappa}\left(u_{-1 / 2}, u_{L}\right) \geq f\left(u_{L}\right)-f(\kappa)=\Phi_{\kappa}\left(u_{L}\right)$.

We now state the straightforward corollary, obtained by subtracting (46) to (44) and (47) to (45). 
Corollary 4.3 For all $\kappa \in[0,1]$,

$$
\begin{gathered}
\frac{\left|u_{-1 / 2}^{n+1}-\kappa\right|-\left|u_{-1 / 2}^{n}-\kappa\right|}{k} h+\Phi_{\kappa}\left(u_{L}^{n}\right)-\Phi_{\kappa}\left(u_{-3 / 2}^{n}, u_{-1 / 2}^{n}\right) \leq 0, \\
\frac{\left|u_{1 / 2}^{n+1}-\kappa\right|-\left|u_{1 / 2}^{n}-\kappa\right|}{k} h+\Phi_{\kappa}\left(u_{1 / 2}^{n}, u_{3 / 2}^{n}\right)-\Phi_{\kappa}\left(u_{R}^{n}\right) \leq 0 .
\end{gathered}
$$

\subsection{Continuous entropy inequalities}

For a $\mathcal{C}^{1}\left(\mathbb{R} \times \mathbb{R}_{+} ; \mathbb{R}\right)$ function $\varphi$, we denote by

$$
\|\nabla \varphi\|=\left\|\partial_{t} \varphi\right\|_{\infty}+\left\|\partial_{x} \varphi\right\|_{\infty} .
$$

Recall that when $\varphi$ is compactly supported, i.e. if $\varphi \in \mathcal{C}_{c}^{1}((-R, R) \times[0, T))$, then there exists $C$ depending only on $R, T$ such that

$$
\|\varphi\|_{\infty} \leq C\|\nabla \varphi\| .
$$

Lemma 4.4 Let $T>0$, and let $\varphi \in \mathcal{D}^{+}((-R, R) \times[0, T))$, then there exists $C$ depending only on $u_{0}, f, A$, $B, R$ and $T$ such that, for all $\kappa \in[0,1]$,

$$
\begin{aligned}
& \int_{\mathbb{R}_{+}} \int_{\mathbb{R}_{-}}\left|u_{h}-\kappa\right| \partial_{t} \varphi d x d t+\int_{\mathbb{R}_{-}}\left|u_{0}-\kappa\right| \varphi(\cdot, 0) d x \\
& \quad+\int_{\mathbb{R}_{+}} \int_{\mathbb{R}_{-}} \Phi_{\kappa}\left(u_{h}\right) \partial_{x} \varphi d x d t-\int_{\mathbb{R}_{+}} \Phi_{\kappa}\left(u_{L, h}\right) \varphi(0, \cdot) d t \geq-C\|\nabla \varphi\| h . \\
& \int_{\mathbb{R}_{+}} \int_{\mathbb{R}_{+}}\left|u_{h}-\kappa\right| \partial_{t} \varphi d x d t+\int_{\mathbb{R}_{+}}\left|u_{0}-\kappa\right| \varphi(\cdot, 0) d x \\
& \quad+\int_{\mathbb{R}_{+}} \int_{\mathbb{R}_{+}} \Phi_{\kappa}\left(u_{h}\right) \partial_{x} \varphi d x d t+\int_{\mathbb{R}_{+}} \Phi_{\kappa}\left(u_{R, h}\right) \varphi(0, \cdot) d t \geq-C\|\nabla \varphi\| h .
\end{aligned}
$$

Proof: We only prove (50) since the proof of (51) is similar. Let $\varphi \in \mathcal{D}^{+}((-R, R) \times[0, T))$, we denote by

$$
\varphi_{i}^{n}=\varphi\left(x_{i}, t^{n}\right), \quad \varphi_{i+1 / 2}^{n}=\varphi\left(x_{i+1 / 2}, t^{n}\right), \quad \forall i \in \mathbb{Z}, \forall n \in \mathbb{N} .
$$

Multiplying equations (43) by $k \varphi_{i+1 / 2}^{n+1}$ and (48) by $k \varphi_{-1 / 2}^{n+1}$, then summing on $i<-1$, one obtains after reorganization of the sums,

$$
T_{1}+T_{2}+T_{3}+T_{4} \geq 0,
$$

with

$$
\begin{aligned}
T_{1} & =\sum_{n \in \mathbb{N}} \sum_{i<0}\left|u_{i+1 / 2}^{n}-\kappa\right|\left(\varphi_{i+1 / 2}^{n+1}-\varphi_{i+1 / 2}^{n}\right) h+\sum_{i<0}\left|u_{i+1 / 2}^{0}-\kappa\right| \varphi_{i+1 / 2}^{0} h, \\
T_{2} & =\sum_{n \in \mathbb{N}} k \sum_{i<0} \Phi_{\kappa}\left(u_{i-1 / 2}^{n}, u_{i+1 / 2}^{n}\right)\left(\varphi_{i+1 / 2}^{n+1}-\varphi_{i-1 / 2}^{n+1}\right) h, \\
T_{3} & =-\sum_{n \in \mathbb{N}} k \Phi_{\kappa}\left(u_{L}^{n}\right) \varphi_{0}^{n+1}, \\
T_{4} & =-\sum_{n \in \mathbb{N}} k \Phi_{\kappa}\left(u_{L}^{n}\right)\left(\varphi_{i-1 / 2}^{n+1}-\varphi_{0}^{n+1}\right) .
\end{aligned}
$$

Firstly, it is easy to check that

$$
\left|T_{4}\right| \leq C\|\nabla \varphi\| h,
$$

and that

$$
\left|T_{3}+\int_{\mathbb{R}_{+}} \Phi_{\kappa}\left(u_{L, h}\right) \varphi(0, \cdot) d t\right| \leq C\|\nabla \varphi\| h,
$$


It follows from Propositions 3.9 and 3.11 (we use here classical computations that we can deduce for example from [12]) and the CFL condition (15) that

$$
\begin{gathered}
\left|T_{1}-\int_{\mathbb{R}_{+}} \int_{\mathbb{R}_{-}}\right| u_{h}-\kappa\left|\partial_{t} \varphi d x d t-\int_{\mathbb{R}_{-}}\right| u_{0}-\kappa|\varphi(\cdot, 0) d x| \leq C h\|\nabla \varphi\|, \\
\left|T_{2}-\int_{\mathbb{R}_{+}} \int_{\mathbb{R}_{-}} \Phi_{\kappa}\left(u_{h}\right) \partial_{x} \varphi d x d t\right| \leq C h\|\nabla \varphi\| .
\end{gathered}
$$

Then (50) follows from (52)-(56).

As a direct consequence of Lemma 4.4, following the idea of R. Eymard et al. [15], exploited by F. Bouchut and B. Perthame [8], we can state the following proposition.

Proposition 4.5 There exist positive local Radon measures $\mu_{L, h}, \mu_{R, h}$ belonging to $\left(\mathcal{C}_{c}\left(\mathbb{R} \times \mathbb{R}_{+}\right)\right)^{\prime}$ such that there exists $C$ depending only on $R, L_{f}$

$$
\mu_{L, h}\left(\omega_{R}\right) \leq C h, \quad \mu_{R, h}\left(\omega_{R}\right) \leq C h,
$$

and such that, for all $\varphi \in \mathcal{D}^{+}\left(\mathbb{R} \times \mathbb{R}_{+}\right)$, one has

$$
\begin{aligned}
& \int_{\mathbb{R}_{+}} \int_{\mathbb{R}_{-}}\left|u_{h}-\kappa\right| \partial_{t} \varphi d x d t+\int_{\mathbb{R}_{-}}\left|u_{0}-\kappa\right| \varphi(\cdot, 0) d x \\
& \quad+\int_{\mathbb{R}_{+}} \int_{\mathbb{R}_{-}} \Phi_{\kappa}\left(u_{h}\right) \partial_{x} \varphi d x d t-\int_{\mathbb{R}_{+}} \Phi_{\kappa}\left(u_{L, h}\right) \varphi(0, \cdot) d t \geq-\left\langle\mu_{L, h},\left|\partial_{t} \varphi\right|+\left|\partial_{x} \varphi\right|\right\rangle, \\
& \int_{\mathbb{R}_{+}} \int_{\mathbb{R}_{+}}\left|u_{h}-\kappa\right| \partial_{t} \varphi d x d t+\int_{\mathbb{R}_{+}}\left|u_{0}-\kappa\right| \varphi(\cdot, 0) d x \\
& \quad+\int_{\mathbb{R}_{+}} \int_{\mathbb{R}_{+}} \Phi_{\kappa}\left(u_{h}\right) \partial_{x} \varphi d x d t+\int_{\mathbb{R}_{+}} \Phi_{\kappa}\left(u_{R, h}\right) \varphi(0, \cdot) d t \geq-\left\langle\mu_{R, h},\left|\partial_{t} \varphi\right|+\left|\partial_{x} \varphi\right|\right\rangle .
\end{aligned}
$$

In section 5, we will use the doubling variable technique introduced by S. N. KRǓZKOv [19] and adapted to this frame by N. N. KuzNETSOV [20]. For this reason, we will assume that the exact solution $u$ depends on the variable $(y, s)$ instead of $(x, t)$. Since $u$ admits strong traces $u_{L}, u_{R} \in L^{\infty}\left(\mathbb{R}_{+} ;[0,1]\right)$ on $\{y=0\} \times \mathbb{R}_{+}$(either thanks to Proposition 3.12 or to [24]), then $u$ satisfies the following entropy inequalities : $\forall \psi \in \mathcal{D}^{+}\left(\mathbb{R} \times \mathbb{R}_{+}\right)$,

$$
\begin{aligned}
& \int_{\mathbb{R}_{+}} \int_{\mathbb{R}_{-}}|u-\kappa| \partial_{s} \psi d y d s+\int_{\mathbb{R}_{-}}\left|u_{0}-\kappa\right| \psi(\cdot, 0) d y \\
& \quad+\int_{\mathbb{R}_{+}} \int_{\mathbb{R}_{-}} \Phi_{\kappa}(u) \partial_{y} \psi d y d s-\int_{\mathbb{R}_{+}} \Phi_{\kappa}\left(u_{L}\right) \psi(0, \cdot) d s \geq 0, \\
& \int_{\mathbb{R}_{+}} \int_{\mathbb{R}_{+}}|u-\kappa| \partial_{s} \psi d y d s+\int_{\mathbb{R}_{+}}\left|u_{0}-\kappa\right| \psi(\cdot, 0) d y \\
& \quad+\int_{\mathbb{R}_{+}} \int_{\mathbb{R}_{+}} \Phi_{\kappa}(u) \partial_{y} \psi d y d s+\int_{\mathbb{R}_{+}} \Phi_{\kappa}\left(u_{R}\right) \psi(0, \cdot) d s \geq 0 .
\end{aligned}
$$

\section{Proof of Theorem 3}

As mentioned before, the proof of the error estimates is based on the doubling variable technique, introduced by S. N. KRUŽKov [19] for proving the uniqueness of the entropy solution to a multidimensional scalar conservation law and later adapted by N. N. KuzneTsov [20] to derive error estimates on the solutions provided by monotone finite difference schemes. First of all, we need to introduce approximation of the unit. 


\subsection{Approximation of the unit}

Because of the presence of the interface $\{x=0\}$, we need to introduce a family of non-even smoothing kernels $\left(\rho_{\epsilon}\right)_{\epsilon>0}$. It is built as follows. Let $\rho \in \mathcal{D}^{+}(\mathbb{R})$ such that $\operatorname{supp}(\rho) \subset[0,1], \int_{\mathbb{R}} \rho(a) d a=1$ and such that $(x-1 / 2) \rho^{\prime}(x) \leq 0$. Let $\epsilon \in(0,1]$, we denote by $\rho_{\epsilon}(x)=\frac{1}{\epsilon} \rho\left(\frac{x}{\epsilon}\right)$, so that $\operatorname{supp}\left(\rho_{\epsilon}\right) \subset[0, \epsilon]$, and

$$
\int_{\mathbb{R}}\left|\rho_{\epsilon}^{\prime}(a)\right| d a=\frac{2}{\epsilon} \rho(1 / 2) \text {. }
$$

\subsection{The case where $F_{h} \equiv F$}

In this section, we first assume that for almost all $t \in\left[t^{n}, t^{n+1}\right), F(t)=F^{n}$, and thus $\left(u_{L, h}(t), u_{R, h}(t)\right) \in \mathcal{G}^{*}(t)$.

Let $\varphi \in \mathcal{D}^{+}((-R, R) \times[0, T))$, then we define the functions $\xi_{L}, \xi_{R}$ by

$$
\xi_{L}(x, t, y, s)=\varphi(x, t) \rho_{\epsilon}(x-y) \rho_{\eta}(s-t), \quad \xi_{R}(x, t, y, s)=\varphi(x, t) \rho_{\epsilon}(y-x) \rho_{\eta}(s-t),
$$

for some $\epsilon, \eta>0$ to be fixed later, and where $\rho_{\epsilon}\left(\right.$ or $\left.\rho_{\eta}\right)$ is the approximation of the unit introduced in Section 5.1. The functions $\xi_{L}$ and $\xi_{R}$ are built so that

$$
\begin{gathered}
\xi_{L}(x, t, y, 0)=\xi_{R}(x, t, y, 0)=0, \quad \forall(x, y, t) \in \mathbb{R}_{2} \times \mathbb{R}_{+}, \\
\xi_{L}(x, t, 0, s)=0, \quad \forall(x, t, s)
\end{gathered}
$$

Let us choose $\kappa=u(y, s)$ in (58) and integrate on $\mathbb{R}_{-} \times \mathbb{R}_{+}$w.r.t. $(y, s)$, and $\kappa=u_{h}(x, t)$ in $(60)$ and integrate on $\mathbb{R}_{-} \times \mathbb{R}_{+}$w.r.t. $(x, t)$, and then sum both contributions. This provides

$$
D_{1}^{L}+D_{2}^{L}+D_{3}^{L}+D_{4}^{L} \geq D_{5}^{L},
$$

where

$$
\begin{aligned}
D_{1}^{L}= & \int_{\mathbb{R}_{+}} \int_{\mathbb{R}_{-}} \int_{\mathbb{R}_{+}} \int_{\mathbb{R}_{-}}\left|u_{h}(x, t)-u(y, s)\right| \partial_{t} \varphi(x, t) \rho_{\epsilon}(x-y) \rho_{\eta}(s-t) d x d t d y d s, \\
D_{2}^{L}= & \int_{\mathbb{R}_{+}} \int_{\mathbb{R}_{-}} \int_{\mathbb{R}_{-}}\left|u_{0}(x)-u(y, s)\right| \varphi(x, 0) \rho_{\epsilon}(x-y) \rho_{\eta}(s) d x d y d s, \\
D_{3}^{L}= & \int_{\mathbb{R}_{+}} \int_{\mathbb{R}_{-}} \int_{\mathbb{R}_{+}} \int_{\mathbb{R}_{-}} \Phi_{u(y, s)}\left(u_{h}(x, t)\right) \partial_{x} \varphi(x, t) \rho_{\epsilon}(x-y) \rho_{\eta}(s-t) d x d t d y d s, \\
D_{4}^{L}= & -\int_{\mathbb{R}_{+}} \int_{\mathbb{R}_{-}} \int_{\mathbb{R}_{+}} \Phi_{u(y, s)}\left(u_{L, h}(t)\right) \varphi(0, t) \rho_{\epsilon}(-y) \rho_{\eta}(s-t) d t d y d s, \\
D_{5}^{L}= & -\int_{\mathbb{R}_{+}} \int_{\mathbb{R}_{-}}\left\langle\mu_{L, h},\left(\left|\partial_{t} \varphi\right|+\left|\partial_{x} \varphi\right|\right) \rho_{\epsilon}(\cdot-y) \rho_{\eta}(s-\cdot)\right\rangle d y d s \\
& -\int_{\mathbb{R}_{+}} \int_{\mathbb{R}_{-}}\left\langle\mu_{L, h}, \varphi\left(\left|\rho_{\epsilon}^{\prime}(\cdot-y)\right| \rho_{\eta}(s-\cdot)+\rho_{\epsilon}(\cdot-y)\left|\rho_{\eta}^{\prime}(s-\cdot)\right|\right)\right\rangle d y d s
\end{aligned}
$$

Among the above terms, only $D_{4}^{L}$ is original in the sense that its treatment has not already been performed in the already mentioned works $[20,17,16]$. Let us first recall the classical results concerning the other terms.

Concerning the term $D_{5}^{L}$, it follows from Fubini-Tonelli theorem and from estimate (57) that

$$
\int_{\mathbb{R}_{+}} \int_{\mathbb{R}_{-}}\left\langle\mu_{L, h},\left(\left|\partial_{t} \varphi\right|+\left|\partial_{x} \varphi\right|\right) \rho_{\epsilon}(\cdot-y) \rho_{\eta}(s-\cdot)\right\rangle d y d s \leq C h\|\nabla \varphi\| .
$$

On the other hand, thanks to (62), one has

$$
\int_{\mathbb{R}_{+}} \int_{\mathbb{R}_{-}}\left\langle\mu_{L, h}, \varphi\left(\left|\rho_{\epsilon}^{\prime}(\cdot,-y)\right| \rho_{\eta}(s-\cdot)+\rho_{\epsilon}(\cdot-y)\left|\rho_{\eta}^{\prime}(s-\cdot)\right|\right)\right\rangle d y d s \leq C\left(\frac{h}{\epsilon}+\frac{h}{\eta}\right)\|\varphi\|_{\infty},
$$

hence

$$
D_{5}^{L} \geq-C\left(h+\frac{h}{\epsilon}+\frac{h}{\eta}\right)\|\nabla \varphi\|
$$


Let us now consider the term $D_{1}$, for which one has

$$
D_{1}^{L} \leq D_{1,1}^{L}+D_{1,2}^{L}
$$

where

$$
\begin{aligned}
D_{1,1}^{L} & =\int_{\mathbb{R}_{+}} \int_{\mathbb{R}_{-}}\left|u_{h}(x, t)-u(x, t)\right| \partial_{t} \varphi(x, t) d x d t \\
D_{1,2}^{L} & =\int_{\mathbb{R}_{+}} \int_{\mathbb{R}_{-}} \int_{\mathbb{R}_{+}} \int_{\mathbb{R}_{-}}|u(x, t)-u(y, s)|\left|\partial_{t} \varphi(x, t)\right| \rho_{\epsilon}(x-y) \rho_{\eta}(s-t) d x d t d y d s .
\end{aligned}
$$

Using the fact that $u$ belongs to $\mathrm{BV}(\operatorname{supp} \varphi)$ (cf. Proposition 3.12), one thus obtains that

$$
D_{1,2}^{L} \leq C(\epsilon+\eta)\|\nabla \varphi\|,
$$

where $C$ only depends on $\operatorname{supp} \varphi, u_{0}, f, A, B$.

In order to estimate $D_{2}^{L}$, we mimic the method proposed in $[15,12,22]$. Therefore, we choose

$$
(y, s) \mapsto \psi(x, t, y, s)=\varphi(x, 0) \rho_{\epsilon}(x-y) \int_{s}^{\infty} \rho_{\eta}(\tau) d \tau
$$

as test function in (60) for $\kappa=u_{0}(x)$ and integrate w.r.t. $x \in \mathbb{R}_{-}$. This provides that

$$
D_{2}^{L} \leq D_{2,1}^{L}+D_{2,2}^{L}+D_{2,3}^{L}
$$

where

$$
\begin{aligned}
D_{2,1}^{L} & =\int_{\mathbb{R}_{-}} \int_{\mathbb{R}_{-}}\left|u_{0}(y)-u_{0}(x)\right| \varphi(x, 0) \rho_{\epsilon}(x-y) d x d y \\
D_{2,2}^{L} & =-\int_{\mathbb{R}_{-}} \int_{\mathbb{R}_{+}} \int_{\mathbb{R}_{-}} \Phi_{u_{0}(y)}(u(y, s)) \varphi(x, 0) \rho_{\epsilon}^{\prime}(x-y) \int_{s}^{\infty} \rho_{\eta}(\tau) d \tau d y d s d x . \\
D_{2,3}^{L} & =-\int_{\mathbb{R}_{-}} \int_{\mathbb{R}_{+}} \int_{\mathbb{R}_{-}}\left(\Phi_{u_{0}(x)}(u(y, s))-\Phi_{u_{0}(y)}(u(y, s))\right) \varphi(x, 0) \rho_{\epsilon}^{\prime}(x-y) \int_{s}^{\infty} \rho_{\eta}(\tau) d \tau d y d s d x .
\end{aligned}
$$

Since $u_{0} \in \mathrm{BV}\left(\mathbb{R}_{-}\right)$, one has

$$
D_{2,1}^{L} \leq C \epsilon\|\varphi\|_{\infty} .
$$

Recall that $b \mapsto \Phi_{b}(a)$ is $L_{f}$-Lipschitz continuous for all $a \in[0,1]$; besides, $\operatorname{supp}\left(s \mapsto \int_{s}^{\infty} \rho_{\eta}(\tau) d \tau\right) \subset[0, \eta]$ and $0 \leq \int_{s}^{\infty} \rho_{\eta}(\tau) d \tau \leq 1$, which gives

$$
\left|D_{2,3}^{L}\right| \leq L_{f} \eta\|\varphi\|_{\infty} \int_{\mathbb{R}_{-}} \int_{\mathbb{R}_{-}}\left|u_{0}(x)-u_{0}(y)\right|\left|\rho_{\epsilon}^{\prime}(x-y)\right| d x d y .
$$

Now, noting that $a \mapsto \frac{\epsilon\left|\rho_{\epsilon}^{\prime}(a)\right|}{2 \rho(1 / 2)}$ is an approximation of the unit leads to

$$
\left|D_{2,3}^{L}\right| \leq C \eta\|\varphi\|_{\infty} .
$$

Integrating $D_{2,2}^{L}$ by parts w.r.t. the variable $x$ provides

$$
\begin{aligned}
D_{2,2}^{L}= & \int_{\mathbb{R}_{-}} \int_{\mathbb{R}_{+}} \int_{\mathbb{R}_{-}} \Phi_{u_{0}(y)}(u(y, s)) \partial_{x} \varphi(x, 0) \rho_{\epsilon}(x-y) \int_{s}^{\infty} \rho_{\eta}(\tau) d \tau d y d s d x \\
& +\int_{\mathbb{R}_{+}} \int_{\mathbb{R}_{-}} \Phi_{u_{0}(y)}(u(y, s)) \varphi(0,0) \rho_{\epsilon}(-y) \int_{s}^{\infty} \rho_{\eta}(\tau) d \tau d y d s .
\end{aligned}
$$

Using again that $\operatorname{supp}\left(s \mapsto \int_{s}^{\infty} \rho_{\eta}(\tau) d \tau\right) \subset[0, \eta]$ and $0 \leq \int_{s}^{\infty} \rho_{\eta}(\tau) d \tau \leq 1$, one obtains that

$$
\left|D_{2,2}^{L}\right| \leq C \eta\left(\|\varphi\|_{\infty}+\|\nabla \varphi\|\right) .
$$


Concerning $D_{3}^{L}$, one has

$$
D_{3}^{L} \leq D_{3,1}^{L}+D_{3,2}^{L}
$$

with

$$
\begin{aligned}
D_{3,1}^{L} & =\int_{\mathbb{R}_{+}} \int_{\mathbb{R}_{-}} \Phi_{u(x, t)}\left(u_{h}(x, t)\right) \partial_{x} \varphi(x, t) d x d t, \\
D_{3,2}^{L} & =L_{f} \int_{\mathbb{R}_{+}} \int_{\mathbb{R}_{-}} \int_{\mathbb{R}_{+}} \int_{\mathbb{R}_{-}}\left|u(x, t)-u(y, s) \| \partial_{x} \varphi(x, t)\right| \rho_{\epsilon}(x-y) \rho_{\eta}(s-t) d x d t d y d s .
\end{aligned}
$$

Therefore, using again that $u \in \mathrm{BV}_{\text {loc }}\left(\mathbb{R} \times \mathbb{R}_{+}\right)$, we deduce that

$$
D_{3,2}^{L} \leq C(\epsilon+\eta)\|\nabla \varphi\| .
$$

The treatment of $D_{4}^{L}$ is quite similar to the treatment of $D_{2}^{L}$. Indeed, choosing

$$
(y, s) \mapsto \varphi(0, t) \rho_{\eta}(s-t) \int_{-y}^{\infty} \rho_{\epsilon}(a) d a
$$

as test function in (60) and integrating for $t \in \mathbb{R}_{+}$yield

$$
D_{4}^{L} \leq D_{4,1}^{L}+D_{4,2}^{L}
$$

where

$$
\begin{aligned}
D_{4,1}^{L} & =-\int_{\mathbb{R}_{+}} \int_{\mathbb{R}_{+}} \Phi_{u_{L}(s)}\left(u_{L, h}(t)\right) \varphi(0, t) \rho_{\eta}(s-t) d t d s \\
D_{4,2}^{L} & =\int_{\mathbb{R}_{+}} \int_{\mathbb{R}_{+}} \int_{\mathbb{R}_{-}}\left|u(y, s)-u_{L, h}(t)\right| \varphi(0, t) \rho_{\eta}^{\prime}(s-t) \int_{-y}^{\infty} \rho_{\epsilon}(a) d a d y d s d t .
\end{aligned}
$$

We deduce from the triangle inequality that

$$
D_{4,2}^{L} \leq D_{4,3}^{L}+D_{4,4}^{L}
$$

where

$$
\begin{aligned}
D_{4,3}^{L} & =\int_{\mathbb{R}_{+}} \int_{\mathbb{R}_{+}} \int_{\mathbb{R}_{-}}\left|u(y, s)-u_{L, h}(s)\right| \varphi(0, t) \rho_{\eta}^{\prime}(s-t) \int_{-y}^{\infty} \rho_{\epsilon}(a) d a d y d s d t, \\
D_{4,4}^{L} & =\epsilon \int_{\mathbb{R}_{+}} \int_{\mathbb{R}_{+}}\left|u_{L, h}(t)-u_{L, h}(s)\right|\left|\rho_{\eta}^{\prime}(s-t)\right| d s d t .
\end{aligned}
$$

Integrating $D_{4,3}^{L}$ by parts w.r.t. the variable $t$ leads to

$$
D_{4,3}^{L}=\int_{\mathbb{R}_{+}} \int_{\mathbb{R}_{-}}\left|u(y, s)-u_{L, h}(s)\right|\left(\int_{-y}^{\infty} \rho_{\epsilon}(a) d a\right)\left\{\varphi(0,0) \rho_{\eta}(s)+\int_{0}^{\infty} \partial_{t} \varphi(0, t) \rho_{\eta}(s-t) d t\right\} d y d s .
$$

Since $0 \leq u(y, s), u_{L, h}(t) \leq 1$, since, $y \mapsto \int_{-y}^{\infty} \rho_{\epsilon}(a) d a$ is compactly supported in $[-\epsilon, 0]$ and bounded by 1 , we obtain that

$$
D_{4,3}^{L} \leq C \epsilon\left(\|\varphi\|_{\infty}+\|\nabla \varphi\|\right) .
$$

We provide now two estimates for $D_{4,4}^{L}$ according to the regularity of $t \mapsto u_{L, h}(t)$.

- First, we do not assume that Assumption 2 holds, then, using the fact that $0 \leq u_{L, h} \leq 1$ a.e. in $\mathbb{R}_{+}$, one has

$$
D_{4,4}^{L} \leq C \frac{\epsilon}{\eta}\|\nabla \varphi\|
$$

- Assume now that Assumption 2 holds, then one obtains that

$$
D_{4,4}^{L} \leq 2 \epsilon \rho(1 / 2) C_{\mathrm{BV}}\|\nabla \varphi\|
$$


Thus it follows from (65)-(79) that

$$
\begin{gathered}
\int_{\mathbb{R}_{+}} \int_{\mathbb{R}_{-}}\left|u_{h}(x, t)-u(x, t)\right| \partial_{t} \varphi(x, t) d x d t+\int_{\mathbb{R}_{+}} \int_{\mathbb{R}_{-}} \Phi_{u(x, t)}\left(u_{h}(x, t)\right) \partial_{x} \varphi(x, t) d x d t \\
-\int_{\mathbb{R}_{+}} \int_{\mathbb{R}_{+}} \Phi_{u_{L}(s)}\left(u_{L, h}(t)\right) \varphi(0, t) \rho_{\eta}(s-t) d t d s \geq-C\|\nabla \varphi\| \Theta(h, \epsilon, \eta),
\end{gathered}
$$

where

$$
\Theta(h, \epsilon, \eta)= \begin{cases}h+\epsilon+\eta+\frac{h}{\epsilon}+\frac{h}{\eta} & \text { if Assumption } 2 \text { holds } \\ h+\epsilon+\eta+\frac{h}{\epsilon}+\frac{h}{\eta}+\frac{\epsilon}{\eta} & \text { otherwise. }\end{cases}
$$

Similar calculations carried out for $(x, t, y, s) \in\left(\mathbb{R}_{+}\right)^{4}$ with the test function $\xi_{R}$ yield

$$
\begin{gathered}
\int_{\mathbb{R}_{+}} \int_{\mathbb{R}_{+}}\left|u_{h}(x, t)-u(x, t)\right| \partial_{t} \varphi(x, t) d x d t+\int_{\mathbb{R}_{+}} \int_{\mathbb{R}_{+}} \Phi_{u(x, t)}\left(u_{h}(x, t)\right) \partial_{x} \varphi(x, t) d x d t \\
\quad+\int_{\mathbb{R}_{+}} \int_{\mathbb{R}_{+}} \Phi_{u_{R}(s)}\left(u_{R, h}(t)\right) \varphi(0, t) \rho_{\eta}(s-t) d t d s \geq-C\|\nabla \varphi\| \Theta(h, \epsilon, \eta) .
\end{gathered}
$$

Adding (80) and (82) provides

$$
\begin{aligned}
& \int_{\mathbb{R}_{+}} \int_{\mathbb{R}}\left|u_{h}(x, t)-u(x, t)\right| \partial_{t} \varphi(x, t) d x d t \\
& \quad+\int_{\mathbb{R}_{+}} \int_{\mathbb{R}_{-}} \Phi_{u(x, t)}\left(u_{h}(x, t)\right) \partial_{x} \varphi(x, t) d x d t+R_{\eta, h}(\varphi) \geq-C\|\nabla \varphi\| \Theta(h, \epsilon, \eta),
\end{aligned}
$$

where

$$
R_{\eta, h}(\varphi)=\int_{\mathbb{R}_{+}} \int_{\mathbb{R}_{+}}\left[\Phi_{u_{R}(s)}\left(u_{R, h}(t)\right)-\Phi_{u_{L}(s)}\left(u_{L, h}(t)\right)\right] \varphi(0, t) \rho_{\eta}(s-t) d t d s .
$$

Lemma 5.1 Let $t>0$ and let $\mathcal{G}^{*}(t)$ be the $L^{1}$-dissipative germ introduced in Definition 1.1, then, for all $\left(c_{L}, c_{R}\right) \in \mathcal{G}^{*}(t)$, for all $\left(\kappa_{L}, \kappa_{R}\right) \in[0,1]^{2}$,

$$
\Phi_{\kappa_{R}}\left(c_{R}\right)-\Phi_{\kappa_{L}}\left(c_{L}\right) \leq L_{f} \operatorname{dist}_{1}\left(\left(\kappa_{L}, \kappa_{R}\right), \mathcal{G}^{*}(t)\right)
$$

where

$$
\operatorname{dist}_{1}\left(\left(\kappa_{L}, \kappa_{R}\right), \mathcal{G}^{*}(t)\right)=\min _{\left(a_{L}, a_{R}\right) \in \mathcal{G}^{*}(t)}\left(\left|\kappa_{L}-a_{L}\right|-\left|\kappa_{R}-a_{R}\right|\right)
$$

Proof: Let $\left(a_{L}, a_{R}\right) \in \mathcal{G}^{*}(t)$, then, thanks to the definition of $\mathcal{G}^{*}(t)$, one has

$$
\Phi_{a_{R}}\left(c_{R}\right)-\Phi_{a_{L}}\left(c_{L}\right) \leq 0
$$

Now, since $\kappa \mapsto \Phi_{\kappa}(s)$ is $L_{f}$-Lipschitz continuous for all $s \in[0,1]$, we obtain that

$$
\Phi_{\kappa_{R}}\left(c_{R}\right)-\Phi_{\kappa_{L}}\left(c_{L}\right) \leq L_{f}\left(\left|\kappa_{L}-a_{L}\right|-\left|\kappa_{R}-a_{R}\right|\right)
$$

Since $\mathcal{G}^{*}(t)$ is closed in $[0,1]^{2}$, the above relation thus still holds for the minimum $\left(a_{L}, a_{R}\right) \in \mathcal{G}^{*}(t)$.

Lemma 5.2 There exists $C$ depending only on $f, T, A, B$ such that

$$
R_{\eta, h}(\psi) \leq C \eta\|\psi\|_{\infty}, \quad \forall \psi \in \mathcal{C}_{c}([0, T) ; \mathbb{R})
$$

Proof: Using the fact that $\Phi_{\kappa}(u)=\Phi_{u}(\kappa)$, it follows from Lemma 5.1 that

$$
\Phi_{u_{R}(s)}\left(u_{R, h}(t)\right)-\Phi_{u_{L}(s)}\left(u_{L, h}(t)\right) \leq L_{f} \max \left\{\operatorname{dist}_{1}\left(\left(u_{L}(s), u_{R}(s)\right), \mathcal{G}^{*}(t)\right), \operatorname{dist}_{1}\left(\left(u_{L, h}(t), u_{R, h}(t)\right), \mathcal{G}^{*}(s)\right)\right\} .
$$

Now, its appears clearly that if $\left(u_{L}(s), u_{R}(s)\right) \in \mathcal{G}^{*}(t)$ or $\left(u_{L, h}(t), u_{R, h}(t)\right) \in \mathcal{G}^{*}(s)$, then

$$
\Phi_{u_{R}(s)}\left(u_{R, h}(t)\right)-\Phi_{u_{L}(s)}\left(u_{L, h}(t)\right) \leq 0 .
$$


Assume now that $\left(u_{L}(s), u_{R}(s)\right) \notin \mathcal{G}^{*}(t)$ and $\left(u_{L, h}(t), u_{R, h}(t)\right) \notin \mathcal{G}^{*}(s)$. This implies that either

$$
F(t)>F(s) \quad \text { and } \quad\left(u_{L}(s), u_{R}(s)\right)=(A(s), B(s)),
$$

or

$$
F(t)<F(s) \quad \text { and } \quad\left(u_{L, h}(t), u_{R, h}(t)\right)=(A(t), B(t)) .
$$

In the first case (84), one has

$$
\operatorname{dist}_{1}\left(\left(u_{L}(s), u_{R}(s)\right) ; \mathcal{G}^{*}(t)\right) \leq|A(t)-A(s)|+|B(t)-B(s)|,
$$

while in the second case (85), one has

$$
\operatorname{dist}_{1}\left(\left(u_{L, h}(t), u_{R, h}(t)\right) ; \mathcal{G}^{*}(s)\right) \leq|A(t)-A(s)|+|B(t)-B(s)| .
$$

Hence,

$$
\Phi_{u_{R}(s)}\left(u_{R, h}(t)\right)-\Phi_{u_{L}(s)}\left(u_{L, h}(t)\right) \leq L_{f}(|A(t)-A(s)|+|B(t)-B(s)|) .
$$

Now, for $\psi \in \mathcal{C}_{c}([0, T) ; \mathbb{R})$, recalling that $\operatorname{supp}\left(\rho_{\eta}\right) \subset[0, \eta]$, one has

$$
R_{\eta, h}(\psi) \leq L_{f}\|\psi\|_{\infty}\left(\sup _{\tau \in[0, \eta]} \int_{0}^{T}|A(t+\tau)-A(t)| d t+\sup _{\tau \in[0, \eta]} \int_{0}^{T}|B(t+\tau)-B(t)| d t\right),
$$

thus we obtain

$$
R_{\eta, h}(\psi) \leq L_{f} T V_{[0, T+1]}(A-B) \eta\|\psi\|_{\infty}
$$

Using Lemma 5.2 in (83) provides that

$$
\int_{\mathbb{R}_{+}} \int_{\mathbb{R}}\left|u_{h}(x, t)-u(x, t)\right| \partial_{t} \varphi(x, t) d x d t+\int_{\mathbb{R}_{+}} \int_{\mathbb{R}} \Phi_{u(x, t)}\left(u_{h}(x, t)\right) \partial_{x} \varphi d x d t \leq C\|\nabla \varphi\| \Theta(h, \epsilon, \eta) .
$$

In order to conclude the proof of Theorem 3 , it only remains to choose a convenient $\varphi$, that is

$$
\varphi(x, t)=\left\{\begin{array}{l}
\zeta\left(|x|-L_{f} t\right) \frac{T-t}{T} \text { if }(x, t) \in \mathbb{R} \times[0, T] \\
0 \text { if } t \geq T
\end{array}\right.
$$

where

$$
\zeta(r)=\max (0, \min (1, R+1-r)), \quad \forall r \in \mathbb{R}_{+},
$$

and to notice that choosing $\epsilon=\eta=h^{1 / 2}$ (under Assumption 2) or $\epsilon=h^{2 / 3}$ and $\eta=h^{1 / 3}$ (general case) provides

$$
\min _{\epsilon, \eta} \Theta(h, \epsilon, \eta) \leq \begin{cases}C h^{1 / 2} & \text { if Assumption } 2 \text { holds } \\ C h^{1 / 3} & \text { otherwise. }\end{cases}
$$

\subsection{The general case}

Denote by $\tilde{u}$ the unique solution to the problem corresponding to the constraint $F_{h}$. Then it has been proven previously that

$$
\iint_{\omega_{R}}\left|u_{h}-\tilde{u}\right| d x d t \leq C h^{\alpha} \quad \text { with } \alpha \in\{1 / 2,1 / 3\} .
$$

In order to achieve the proof of Theorem 3, it only remains to show that

$$
\iint_{\omega_{R}}|u-\tilde{u}| d x d t \leq C h^{1 / 2}
$$

In fact, one has a better estimate, thanks to the following Proposition, proved in Appendix of [4]. 
Proposition $5.3([4])$ Let $F, \breve{F} \in L^{\infty}\left(\mathbb{R}_{+} ;[0, f(\bar{u})]\right)$, and let $u, \breve{u}$ be the solutions corresponding respectively to the constraint $F, \breve{F}$ and to a similar initial data $u_{0}$. Then,

$$
\int_{0}^{T} \int_{\mathbb{R}}|u-\breve{u}| d x d t \leq 2 \int_{0}^{T}|F-\breve{F}| d t .
$$

Since $A$ is supposed to belong to $\mathrm{BV}(0, T)$, then $F=f(A)$ also belongs to $\mathrm{BV}(0, T)$. As a consequence, there exists $C$ depending only on $A, f, T$ such that

$$
\left\|F-F_{h}\right\|_{L^{1}(0, T)} \leq C h .
$$

We deduce from the above estimate and from Proposition 5.3 the following corollary that achieves the proof of Theorem 3.

Corollary 5.4 Under Assumption 1, there exists $C$ depending only on $A, f, T$ such that

$$
\iint_{\omega_{R}}|u-\tilde{u}| d x d t \leq C h .
$$

\section{$6 \quad$ Numerical illustration}

We now present some numerical simulations in order to illustrate the error estimate (22). Two conservation laws are investigated: the first one is based on the flux (called the hat flux in the following)

$$
f(u)=1 / 2-|u-1 / 2|
$$

which has the particularity of having linear two parts and the second one is based on the flux (called the GNL flux - as genuinely non linear - in the following)

$$
f(u)=u(1-u)
$$

which is strictly concave. While the present work is devoted to the analysis of the Godunov scheme, we also present the results obtained with the Rusanov scheme:

$$
G(a, b)=\frac{f(a)+f(b)}{2}-\frac{\max \left(\left|f^{\prime}(a)\right|,\left|f^{\prime}(b)\right|\right)}{2}(b-a)
$$

and the constraint is still handled using the trick (14). The initial data for the test case is

$$
u(x, 0)= \begin{cases}0.4 & \text { if }-1 / 2 \leq x<0, \\ 0.5 & \text { if } 0 \leq x \leq 1 / 2,\end{cases}
$$

and the final time is 0.3. For each flux, the constraint is set to $F=0.2$ and is activated (see Figure 2, left). For the hat flux, the solution is composed of a left-going shock wave, a nonclassical stationary shock and a right-going linear wave. For the GNL flux, the solution is composed of a left-going shock wave, a nonclassical stationary shock and a right-going shock wave. The rates of convergence are displayed in Figure 2, right. They are the same for both numerical schemes, which let us think that our result should be extended for any monotone numerical scheme. For the hat flux, the measured rate is $1 / 2$ (and therefore it attests the optimality of our result) while the measured rate is 1 for the GNL flux. Note that in the latter case, it means that the constraint does not alter the classical rate of convergence.

\section{Concluding remarks}

\subsection{A posteriori error estimate}

As noticed by D. KRÖNER and M. OHLBERGER [18], the doubling variable approach used for obtaining error estimates provides a posteriori estimators, i.e. that for all compact subset $K$ of $\mathbb{R} \times \mathbb{R}_{+}$, there exists $\eta_{K}$ depending only $f, K, u_{0}, A, B$ (but not on the exact solution $u$ ) such that

$$
\iint_{K}\left|u_{h}(x, t)-u(x, t)\right| d x d t \leq \eta_{K}\left(u_{h}\right) .
$$



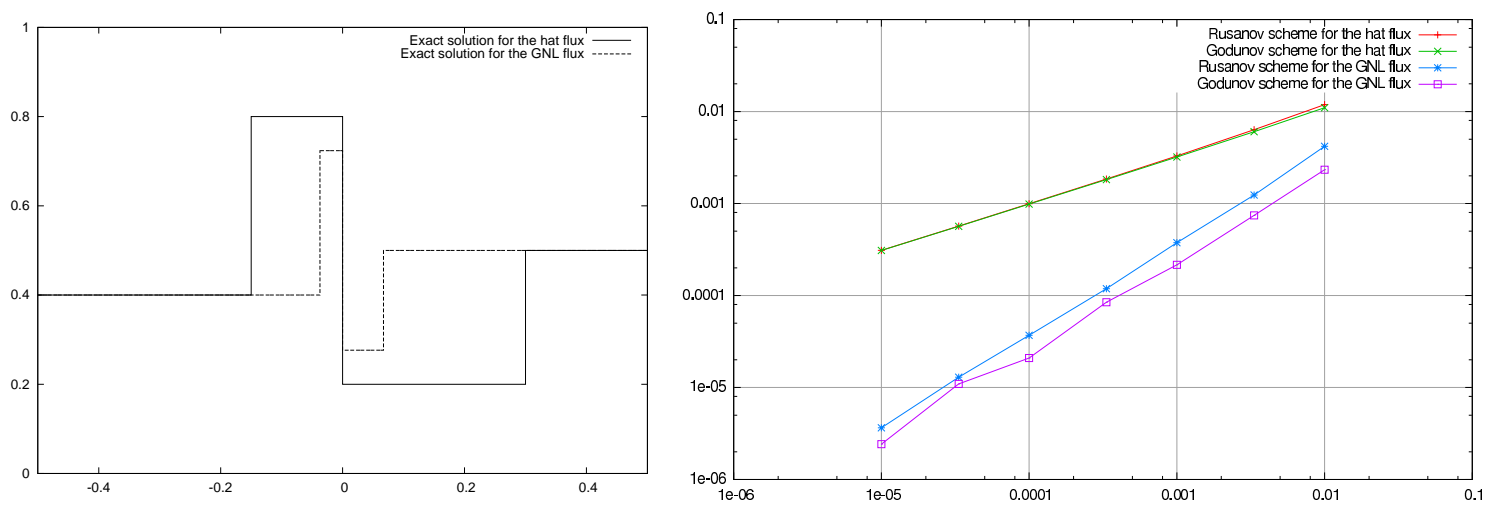

Figure 2: Left: Exact solutions for each flux ( $u$ vs $x$ ). Right: Rates of convergence for each flux and each numerical scheme ( $L^{1}$ error vs $h$ in Log-scale)

Since the right-hand side in the above estimate is fully computable, this permits the localization of the error, and an adaptive mesh refinement strategy (we refer to [18] for more details on both the derivation of the $a$ posteriori estimator and the mesh refinement algorithm). As a consequence, our estimates can be used to develop a posteriori estimators for constrained conservation laws.

\subsection{Comments on the optimality of the result}

The order $h^{1 / 2}$ is optimal in the sense that it can be recovered in some particular cases. Indeed, choosing $f(u)=1 / 2-|u-1 / 2|, F \equiv 1 / 2$ (this means that $A \equiv B \equiv 1 / 2$, so that the constraint is always inactive), and $u_{0}$ in $\mathrm{BV}(\mathbb{R})$ such that $0 \leq u_{0} \leq 1 / 2$. Then the problem turns to be the standard linear equation $\partial_{t} u+\partial_{x} u=0$, and the Godunov scheme becomes the upwind scheme. It is well known that in this case, the error behaves as $h^{1 / 2}$, as illustrated in Figure 2 .

In the case where $f$ is uniformly concave, the numerical experiments provide an error of order $h$.

\subsection{The case of discontinuous flux function}

Consider the case of a scalar conservation law with discontinuous flux function, i.e.

$$
\partial_{t} u+\partial_{x} f(x, u)=0,
$$

where $f(x, u)=f_{L}(u)$ if $x<0$ and $f(x, u)=f_{R}(u)$ if $x>0$, with $f_{L}, f_{R}$ bell-shaped reaching their maximum respectively in $\bar{u}_{L}, \bar{u}_{R}$. As pointed out by ADIMURTHI and VEERAPPA GowDA [2], an infinite number of $L^{1}$-contractive semi-groups can be built for such an equation, and a criterion has to be taken into account in order to select one. We refer to the recent contributions of R. Bürger et al. [9], B. Andreianov et al. [5] and references therein for an overview of this topic, and in particular to the resolution of the Riemann problem arising at the interface, thanks to which we can define the Godunov scheme, and its approximate solution $u_{h}$. In the case where $f_{L} \neq f_{R}$, no BV estimate is available on $u$ (neither on $u_{h}$ ), but we can prove that the Temple function

$$
(x, t) \mapsto \Phi_{\bar{u}}(u(x, t), x):=\left\{\begin{array}{l}
\operatorname{sign}\left(u(x, t)-\bar{u}_{L}\right)\left(f_{L}(u(x, t))-f_{L}\left(\bar{u}_{L}\right)\right) \text { if } x<0, \\
\operatorname{sign}\left(u(x, t)-\bar{u}_{R}\right)\left(f_{R}(u(x, t))-f_{R}\left(\bar{u}_{R}\right)\right) \text { if } x>0
\end{array}\right.
$$

belong to $\mathrm{BV}_{\text {loc }}\left(\mathbb{R} \times \mathbb{R}_{+}\right)$(see e.g. [26, 7, 10]). By the use of numerical diffusion introduced by the scheme (see $[13,27,15,12,22]$ ), it is still possible to derive an error estimate. Indeed, all the tools introduced in the paper, excepted in Section 3, can be adapted to the case of discontinuous flux functions. Nevertheless, the theoretical convergence speed will depend on the continuity modulus of the function $\left(\Phi_{\bar{u}}\right)^{-1}$, and will be furthermore degraded by the fact that no strong BV-estimate is available on the exact solution itself. 


\section{References}

[1] Adimurthi, S. Mishra, and G. D. Veerappa Gowda. Optimal entropy solutions for conservation laws with discontinuous flux-functions. J. Hyperbolic Differ. Equ., 2(4):783-837, 2005.

[2] Adimurthi and G. D. Veerappa Gowda. Conservation law with discontinuous flux. J. Math. Kyoto Univ., $43(1): 27-70,2003$

[3] B. Andreianov and C. Cancès. The Godunov scheme for scalar conservation laws with discontinuous bell-shaped flux functions. To appear in Appl. Math. Lett. (DOI: 10.1016/j.aml.2012.02.044), 2012.

[4] B. Andreianov, P. Goatin, and N. Seguin. Finite volume schemes for locally constrained conservation laws. Numer. Math., 115(4):609-645, 2010. With supplementary material available online.

[5] B. Andreianov, K. H. Karlsen, and N. H. Risebro. A theory of $L^{1}$-dissipative solvers for scalar conservation laws with discontinuous flux. Arch. Ration. Mech. Anal., 201(1):27-86, 2011.

[6] H. Attouch, G. Buttazzo, and G. Michaille. Variational analysis in Sobolev and BV spaces, volume 6 of MPS/SIAM Series on Optimization. Society for Industrial and Applied Mathematics (SIAM), Philadelphia, PA, 2006. Applications to PDEs and optimization.

[7] F. Bachmann. Analysis of a scalar conservation law with a flux function with discontinuous coefficients. Adv. Differential Equations, 9(11-12):1317-1338, 2004.

[8] F. Bouchut and B. Perthame. Kružkov's estimates for scalar conservation laws revisited. Trans. Amer. Math. Soc., 350(7):2847-2870, 1998.

[9] R. Bürger, K. H. Karlsen, and J. D. Towers. An Engquist-Osher-type scheme for conservation laws with discontinuous flux adapted to flux connections. SIAM J. Numer. Anal., 47(3):1684-1712, 2009.

[10] C. Cancès. Asymptotic behavior of two-phase flows in heterogeneous porous media for capillarity depending only on space. I. Convergence to the optimal entropy solution. SIAM J. Math. Anal., 42(2):946-971, 2010 .

[11] C. Cancès and T. Gallouët. On the time continuity of entropy solutions. J. Evol. Equ., 11(1):43-55, 2011.

[12] C. Chainais-Hillairet. Finite volume schemes for a nonlinear hyperbolic equation. Convergence towards the entropy solution and error estimate. M2AN Math. Model. Numer. Anal., 33(1):129-156, 1999.

[13] B. Cockburn, F. Coquel, and P. G. LeFloch. An error estimate for finite volume methods for multidimensional conservation laws. Math. Comp., 63(207):77-103, 1994.

[14] R. M. Colombo and P. Goatin. A well posed conservation law with a variable unilateral constraint. $J$. Differential Equations, 234(2):654-675, 2007.

[15] R. Eymard, T. Gallouët, M. Ghilani, and R. Herbin. Error estimates for the approximate solutions of a nonlinear hyperbolic equation given by finite volume schemes. IMA J. Numer. Anal., 18(4):563-594, 1998.

[16] R. Eymard, T. Gallouët, and R. Herbin. Finite volume methods. Ciarlet, P. G. (ed.) et al., in Handbook of numerical analysis. North-Holland, Amsterdam, pp. 713-1020, 2000.

[17] E. Godlewski and P.-A. Raviart. Hyperbolic systems of conservation laws, volume 3/4 of Mathématiques E5 Applications (Paris) [Mathematics and Applications]. Ellipses, Paris, 1991.

[18] D. Kröner and M. Ohlberger. A posteriori error estimates for upwind finite volume schemes for nonlinear conservation laws in multidimensions. Math. Comp., 69(229):25-39, 2000.

[19] S. N. Kružkov. First order quasilinear equations with several independent variables. Mat. Sb. (N.S.), 81 (123):228-255, 1970.

[20] N. N. Kuznetsov. Accuracy of some approximate methods for computing the weak solutions of a firstorder quasi-linear equation. USSR Comput. Math. and Math. Phys., 16:105-119, 1976. 
[21] M. J. Lighthill and G. B. Whitham. On kinematic waves. II. A theory of traffic flow on long crowded roads. Proc. Roy. Soc. London. Ser. A., 229:317-345, 1955.

[22] M. Ohlberger and J. Vovelle. Error estimate for the approximation of nonlinear conservation laws on bounded domains by the finite volume method. Math. Comp., 75(253):113-150 (electronic), 2006.

[23] O. A. Oleŭnik. Discontinuous solutions of non-linear differential equations. Amer. Math. Soc. Transl. (2), 26:95-172, 1963.

[24] E. Yu. Panov. Existence of strong traces for quasi-solutions of multidimensional conservation laws. J. Hyperbolic Differ. Equ., 4(4):729-770, 2007.

[25] P. I. Richards. Shock waves on the highway. Operations Res., 4:42-51, 1956.

[26] N. Seguin and J. Vovelle. Analysis and approximation of a scalar conservation law with a flux function with discontinuous coefficients. Math. Models Methods Appl. Sci., 13(2):221-257, 2003.

[27] J.-P. Vila. Convergence and error estimates in finite volume schemes for general multidimensional scalar conservation laws. I. Explicit monotone schemes. RAIRO Modél. Math. Anal. Numér., 28(3):267-295, 1994.

[28] A. I. Vol'pert. Spaces BV and quasilinear equations. Mat. Sb. (N.S.), 73 (115):255-302, 1967. 